\begin{tabular}{|c|l|}
\hline Title & $\begin{array}{l}\text { Dust ring formation due to sublimation of dust grains drifting radially inward by the Poynting-Robertson drag: An } \\
\text { analytical model }\end{array}$ \\
\hline Author(s) & Kobay ashi, Hiroshi; W atanabe, Sei-ichiro; Kimura, Hiroshi; Y amamoto, Tetsuo \\
\hline Citation & $\begin{array}{l}\text { Icarus, 201(1), 395-405 } \\
\text { https://doi.org/10.1016/.icarus.2009.01.002 }\end{array}$ \\
\hline Issue Date & $2009-05$ \\
\hline Doc URL & http://hdl.handle.net/2115/38550 \\
\hline Type & article (author version) \\
\hline File Information & 201-1_p395-405.pdf \\
\hline
\end{tabular}

Instructions for use 


\title{
Dust Ring Formation due to Sublimation of Dust Grains Drifting Radially Inward by the Poynting-Robertson Drag: An Analytical Model
}

\author{
Hiroshi Kobayashi $^{1,2}$, Sei-ichiro Watanabe ${ }^{2}$, Hiroshi Kimura $^{1}$ and Tetsuo Yamamoto ${ }^{1}$ \\ ${ }^{1}$ Institute of Low Temperature Science, Hokkaido University \\ Institute of Low Temperature Science, Hokkaido University, Kita-Ku Kita 19 Nishi 8, \\ Sapporo 060-0819, JAPAN \\ ${ }^{2}$ Department of Earth and Planetary Sciences, Graduate School of Environmental Studies, \\ Nagoya University \\ Furo-cho, Chikusa-ku, Nagoya, 464-8601, JAPAN \\ hkobayas@lowtem.hokudai.ac.jp
}

Manuscript pages: 48

Figures: 6

Tables: 2 
Running head: An Analytical Model of Sublimation Dust Ring Formation

Correspondence Address:

Hiroshi Kobayashi

Institute of Low Temperature Science, Hokkaido University

Kita-Ku Kita 19 Nishi 8, Sapporo 060-0819, JAPAN

Tel: $+81-11-706-5486$

Fax: +81-11-706-7142

hkobayas@lowtem.hokudai.ac.jp 


\begin{abstract}
Dust particles exposed to the stellar radiation and wind drift radially inward by the Poynting-Robertson (P-R) drag and pile up at the zone where they begin to sublime substantially. The reason they pile up or form a ring is that their inward drifts due to the P-R drag are suppressed by stellar radiation pressure when the ratio of radiation pressure to stellar gravity on them increases during their sublimation phases. We present analytic solutions to the orbital and mass evolution of such subliming dust particles, and find their drift velocities at the pileup zone are almost independent of their initial semimajor axes and masses. We derive analytically an enhancement factor of the number density of the particles from their drift velocities at the outer edge of the sublimation zone. We show that the formula of the enhancement factor reproduces well numerical simulations in the previous studies. The enhancement factor for spherical dust particles of silicate and carbon extends from 3 to more than 20 at stellar luminosities $L_{\star}=0.8-500 L_{\odot}$, where $L_{\odot}$ is solar luminosity. Although the enhancement factor for fluffy dust particles is smaller than that for spherical particles, sublimating particles inevitably form a dust ring as long as their masses decrease faster than their surface areas during sublimation. The formulation is applicable to dust ring formation for arbitrary shape and material of dust in dust-debris disks as well as in the Solar System.
\end{abstract}


Key Words: Celestial Mechanics, Interplanetary Dust, Debris Disk 


\section{INTRODUCTION}

An accumulation of interplanetary dust grains at their sublimation zone proposed by Belton (1966) is now known as a mechanism to form a dust ring. Refractory dust grains drift toward the Sun by the Poynting-Robertson drag (hereafter P-R drag) and sublime in the immediate vicinity of the Sun. Because the particles lose mass during sublimation, the ratio of radiation pressure to gravity of the Sun ordinarily increases. As a result, their radial drift rates decelerate and the particles pile up at the sublimation zone (e.g., Mukai and Yamamoto 1979; Burns et al. 1979).

In steady state, the number density of dust grains is determined by their inward mass flux due to the P-R drag and is inversely proportional to distance from the central star, irrespective of their shapes and materials. The location of the sublimation zone and the enhancement factor of the number density at the sublimation zone, however, depend on the dust shapes and materials through their optical and thermodynamical properties. Because the shape and material dependences are highly complex, previous studies were conducted for specific shapes and materials without generalization of the results. These studies show that the number density is enhanced by a factor of five due to sublimation for spherical silicate grains and ten for carbon spheres at a few solar radii from the Sun (Mukai and Yamamoto 1979). The results appear to be significantly different if one deals with dust grains having highly porous structures (Kimura et al. 1997). Icy dust grains also pile up at the ice sublimation zone in dust-debris disks around main-sequence stars. The 
number-density enhancement factor is about three at $20-40 \mathrm{AU}$ for $L_{\star} \lesssim 30 L_{\odot}$ and more than 20 at $65\left(L_{\star} / 100 L_{\odot}\right)^{1 / 2} \mathrm{AU}$ for $L_{\star} \gtrsim 30 L_{\odot}$, (Kobayashi et al. 2008).

Analytical solutions to the equation of motion for subliming dust in electromagnetic radiation and weak gravitational fields of a star have not yet been found. Therefore, the previous studies were carried out by numerical integrations of the equation of motion along with the mass loss rate by sublimation. In view of the rapid development of observation facilities to directly image debris disks around main-sequence stars, we need a model that allows us a comparative study of the orbital and mass evolution (including pile-up) of dust grains in the disks without carrying out numerical simulations for specific dust parameters such as material, shape, and structure. This paper presents analytical solutions to orbital and mass evolution of subliming grains in nearly circular orbits valid to the first order in eccentricity in the elliptical expansions. We also derive steady-state analytic formulae for the location and concentration of a dust ring as functions of the optical and thermodynamical properties of the grains. In $\S 2$, we describe equations of motion and a sublimation rate. In $\S 3.1$, we develop a formulation of orbital-averaged temporal differentiation of orbital elements and masses of dust grains under the influence of P-R drag and sublimation. Because we are concerned with the orbital and mass evolution of dust grains with low eccentricities, the orbital-averaged temporal differentiations are expanded in the orbital eccentricity in $§ 3.2$. For the evolution of the orbital elements and dust mass, we derive analytic approximations accurate to the first order in the eccentricity in $\S 3.3$. The enhancement factors of the number density and the optical depth for dust grains are given 
in $§ 3.4$. Our main results are summarized in $\S 4$ with a crude application of the formulae to given systems.

\section{BASIC EQUATIONS}

\subsection{EQUATION OF MOTION}

A solid object that is not separable without external forces is referred to as a dust particle throughout the paper. We do not limit our treatment to compact spherical particles but deal with any shapes and structures of dust by bearing fluffy dust aggregates in mind. We consider the dynamical effects of stellar gravity, radiation, and wind acting on randomly rotating dust particles in orbit around a star. The equation of motion for a dust particle of mass $m$ at position $\boldsymbol{r}$ with respect to the central star of mass $M_{\star}$ is given (e.g., Burns et al. 1979) by

$$
\frac{d^{2} \boldsymbol{r}}{d t^{2}}=-\frac{G M_{\star}}{r^{2}}\left[\left(1-\beta_{\text {total }}\right) \hat{\boldsymbol{r}}+\beta_{\mathrm{PR}}\left(\frac{2 v_{r}}{c} \hat{\boldsymbol{r}}+\frac{v_{\theta}}{c} \hat{\boldsymbol{\theta}}\right)\right]
$$

where $r=|\boldsymbol{r}|, \hat{\boldsymbol{r}}=\boldsymbol{r} / r, \hat{\boldsymbol{\theta}}$ is the unit vector perpendicular to $\boldsymbol{r}$ in the particle's orbital plane, $v_{r}$ and $v_{\theta}$ are the $\hat{\boldsymbol{r}}$ and $\hat{\boldsymbol{\theta}}$ components of the velocity of the particle, $G$ is the gravitational constant, $c$ is the speed of light, $\beta_{\text {total }}$ is the ratio of the radial force due to direct pressures from both stellar radiation and wind to the stellar gravitational force, and $\beta_{\mathrm{PR}}$ is the coefficient of $\mathrm{P}-\mathrm{R}$ drag from the stellar radiation and the stellar wind. The first term in the brackets represents the sum of the gravitational force and the pressure force due to the stellar radiation and wind and the second term represents the P-R drag from the 
stellar radiation and wind. We call the term involving $\beta_{\text {total }}$ in Eq. (1) the pressure force and the term involving $\beta_{\mathrm{PR}}$ the P-R drag.

The ratio $\beta_{\text {total }}$ is expressed by

$$
\beta_{\text {total }}=\beta+\beta_{\mathrm{sw}}
$$

where $\beta$ is the ratio of the electromagnetic radiation pressure to the gravity force and $\beta_{\mathrm{sw}}$ is the ratio of the stellar wind pressure to the gravitational force (e.g., Burns et al. 1979). Using momentum-transfer cross-sections of particles of mass $m$ from the stellar radiation, $C_{\mathrm{pr}}$, and from the stellar wind, $C_{\mathrm{sw}}$, the ratios $\beta$ and $\beta_{\mathrm{sw}}$ are given by (Gustafson 1994; Minato et al. 2004; 2006),

$$
\beta=\frac{L_{\star} \bar{C}_{\mathrm{pr}}}{4 \pi c G M_{\star} m}, \quad \beta_{\mathrm{sw}}=\frac{\dot{M}_{\star} \bar{v}_{\mathrm{sw}} \bar{C}_{\mathrm{sw}}}{4 \pi G M_{\star} m}
$$

where $\dot{M}_{\star}$ is the stellar mass-loss rate by the stellar wind. Here, $\bar{C}_{\mathrm{pr}}$ is the radiation-pressure cross-section $C_{\mathrm{pr}}(\lambda, m)$ averaged over the stellar radiation spectrum and is given by

$$
\bar{C}_{\mathrm{pr}}(m)=\frac{4 \pi R_{\star}^{2}}{L_{\star}} \int_{0}^{\infty} B_{\star}(\lambda) C_{\mathrm{pr}}(\lambda, m) d \lambda,
$$

where $B_{\star}(\lambda)$ is the stellar radiance at a wavelength of $\lambda$ and $R_{\star}$ is the stellar radius.

Similarly, $\bar{C}_{\mathrm{sw}}$ is the momentum-transfer cross-section $C_{\mathrm{sw}}\left(v_{\mathrm{sw}}, m\right)$ averaged over the stellar wind velocity distribution and is given by

$$
\bar{C}_{\mathrm{sw}}(m)=\frac{1}{\bar{v}_{\mathrm{sw}}} \int f_{\mathrm{sw}}\left(v_{\mathrm{sw}}\right) C_{\mathrm{sw}}\left(v_{\mathrm{sw}}, m\right) v_{\mathrm{sw}} d v_{\mathrm{sw}}
$$

where $\bar{v}_{\mathrm{sw}}$ is the mean radial velocity of stellar wind, $v_{\mathrm{sw}}$, given by

$$
\bar{v}_{\mathrm{sw}}=\int_{0}^{\infty} v_{\mathrm{sw}} f_{\mathrm{sw}}\left(v_{\mathrm{sw}}\right) d v_{\mathrm{sw}}
$$


with $f_{\mathrm{sw}}\left(v_{\mathrm{sw}}\right)$ being the distribution function of radial velocities of stellar wind normalized such that

$$
\int_{0}^{\infty} f_{\mathrm{sw}}\left(v_{\mathrm{sw}}\right) d v_{\mathrm{sw}}=1
$$

The coefficient of the P-R drag, $\beta_{\mathrm{PR}}$, is given by (Minato et al. 2004; 2006)

$$
\beta_{\mathrm{PR}}=\beta(1+\gamma)
$$

where

$$
\gamma=\frac{\dot{M}_{\star} c^{2}}{L_{\star}} \frac{\bar{C}_{\mathrm{sw}}}{\bar{C}_{\mathrm{pr}}}
$$

is the ratio of the stellar-wind P-R drag to the stellar-radiation P-R drag. The value of $\gamma$ is evaluated to be

$$
\gamma=0.3\left(\frac{\dot{M}_{\star}}{\dot{M}_{\odot}}\right)\left(\frac{L_{\star}}{L_{\odot}}\right)^{-1}\left(\frac{\bar{C}_{\mathrm{sw}}}{\bar{C}_{\mathrm{pr}}}\right)
$$

implying that the stellar wind drag is important unless $L_{\star} / \dot{M}_{\star} \gg L_{\odot} / \dot{M}_{\odot}$, where $\dot{M}_{\odot}$ is the mass loss rate of the present Sun by the solar wind (Minato et al. 2006). On the other hand, the ratio of the wind to radiation pressure forces is evaluated to be

$$
\frac{\beta_{\mathrm{sw}}}{\beta}=5 \times 10^{-4}\left(\frac{\dot{M}_{\star}}{\dot{M}_{\odot}}\right)\left(\frac{L_{\star}}{L_{\odot}}\right)^{-1}\left(\frac{\bar{C}_{\mathrm{sw}}}{\bar{C}_{\mathrm{pr}}}\right)\left(\frac{\bar{v}_{\mathrm{sw}}}{4.5 \times 10^{7} \mathrm{~cm} \mathrm{~s}^{-1}}\right) .
$$

The stellar wind pressure is ineffective unless $\left(\dot{M}_{\star} / \dot{M}_{\odot}\right)\left(L_{\star} / L_{\odot}\right)^{-1} \gtrsim 10^{3}$.

\subsection{SUBLIMATION RATE}

Particles drift radially inward from an outer region of a circumstellar disk by the P-R drag and then sublime at a certain distance from the star. When they lose mass by 
sublimation, the mass sublimation rate for a particle with its total surface area $A$ is given by

$$
-\frac{d m}{d t}=A P_{\mathrm{v}}(T) \sqrt{\frac{\mu m_{\mathrm{u}}}{2 \pi k T}},
$$

where $\mu$ is the mean molecular weight of the dust material, $m_{\mathrm{u}}$ is the atomic mass unit, and $k$ is the Boltzmann constant. We neglect other mass-loss mechanisms such as sputtering by stellar wind and UV radiation. The saturated vapor pressure $P_{\mathrm{v}}$ at the dust temperature $T$ is expressed by

$$
P_{\mathrm{v}}(T)=P_{0}(T) \exp \left(-\frac{\mu m_{\mathrm{u}} H}{k T}\right),
$$

where $P_{0}(T)$ is only weakly dependent on $T$ and $H$ is the latent heat of sublimation.

The temperature $T$ of a dust particle is determined by energy balance among absorption of incident stellar radiation, emission of thermal radiation, and the latent heat of sublimation in general. However, even in the region where sublimation starts substantially (Stage II, see $§ 3.2$ ), the energy released by sublimation is much smaller than the energy absorbed from the central star and the energy emitted as thermal radiation (Mukai and Yamamoto 1979). Hence, the equation of energy balance can be approximated by

$$
\left[1-\sqrt{1-\left(\frac{R_{\star}}{r}\right)^{2}}\right] \frac{L_{\star} \bar{C}_{\star}}{2 \pi R_{\star}^{2}}=4 \bar{C}_{\mathrm{d}} \sigma_{\mathrm{SB}} T^{4},
$$

where $\sigma_{\mathrm{SB}}$ is the Stefan-Boltzmann constant and $\bar{C}_{\star}$ and $\bar{C}_{\mathrm{d}}(T, m)$ are its mean absorption cross-section for stellar radiation flux and its Planck mean absorption cross-section with $T$, respectively, given by

$$
\bar{C}_{\star}(m)=\frac{4 \pi R_{\star}^{2}}{L_{\star}} \int_{0}^{\infty} B_{\star}(\lambda) C_{\mathrm{abs}}(\lambda, m) d \lambda,
$$




$$
\bar{C}_{\mathrm{d}}(T, m)=\frac{1}{\sigma_{\mathrm{SB}} T^{4}} \int_{0}^{\infty} B(\lambda, T) C_{\mathrm{abs}}(\lambda, m) d \lambda
$$

where $C_{\text {abs }}(\lambda, m)$ is the absorption cross section of a particle with mass $m$ at a wavelength of $\lambda$ and $B(\lambda, T)=2 \pi h c^{2} \lambda^{-5}[\exp (h c / \lambda k T)-1]^{-1}$ is the Planck function at a wavelength of $\lambda$ for the dust temperature $T$ with $h$ being the Planck's constant.

\section{ANALYTICAL CALCULATIONS}

We deal with dust of arbitrary shape and structure as mentioned previously and derive formulae applicable to study its orbital and mass evolution in this section. The formulae for a compact spherical particle are summarized in Appendix A for reference.

\subsection{ORBITAL-AVERAGED CHANGING RATES OF SEMIMAJOR AXIS, ECCENTRICITY, AND MASS}

The osculating elements of semimajor axis $a$ and eccentricity $e$ for an orbit of a dust particle, which are defined in terms of its position $r$ and velocity $v$ at a moment, are given (e.g., Burns et al. 1979) by

$$
\begin{aligned}
a & =\left[\frac{2}{r}-\frac{v^{2}}{\left(1-\beta_{\text {total }}\right) G M_{\star}}\right]^{-1}, \\
e & =\sqrt{1-\frac{r^{2} v_{\theta}^{2}}{\left(1-\beta_{\text {total }}\right) G M_{\star}}\left[\frac{2}{r}-\frac{v^{2}}{\left(1-\beta_{\text {total }}\right) G M_{\star}}\right]} .
\end{aligned}
$$


Differentiating $a$ and $e$ with respect to time $t$ and substituting the terms on the right-hand-side of Eq. (1) for $\dot{\boldsymbol{v}}$, we obtain $d a / d t$ and de/dt (cf. Burns et al. 1979)

$$
\begin{aligned}
\frac{d a}{d t}= & -\eta \frac{\beta_{\text {total }}}{1-\beta_{\text {total }}} \frac{a}{m} \frac{1+2 e \cos f+e^{2}}{1-e^{2}} \frac{d m}{d t} \\
& -\frac{2 \beta_{\mathrm{PR}}}{c\left(1-e^{2}\right)^{3}} \frac{G M_{\star}}{a}\left[1+2 e \cos f+e^{2}\left(1+\sin ^{2} f\right)\right](1+e \cos f)^{2}, \\
\frac{d e}{d t}= & -\eta \frac{\beta_{\text {total }}}{1-\beta_{\text {total }}} \frac{1}{m}(e+\cos f) \frac{d m}{d t} \\
& -\frac{\beta_{\mathrm{PR}}}{c\left(1-e^{2}\right)^{2}} \frac{G M_{\star}}{a^{2}}\left[e\left(2+\sin ^{2} f\right)+2 \cos f\right](1+e \cos f)^{2},
\end{aligned}
$$

where $f$ is the true anomaly and

$$
\eta=-\frac{d \ln \beta_{\text {total }}}{d \ln m}
$$

We note that $\eta>0$ if the dust is larger than the wavelength $\lambda_{\star}$ at the maximum stellar radiation spectrum because $\beta_{\text {total }}=\beta$ unless $\left(\dot{M}_{\star} / \dot{M}_{\odot}\right)\left(L_{\star} / L_{\odot}\right)^{-1} \gtrsim 10^{3}$. In this paper, we consider dust particles with $d \beta / d m<0$, which is satisfied by particles with sizes larger than $\lambda_{\star}$. The first terms in Eqs. (19) and (20) represent changes in $a$ and $e$ due to sublimation and the second ones represent those due to the P-R drag.

When a dust particle drifts radially inward from an outer cold region by the P-R drag, the timescales of changes in the orbital elements and the particle mass are much longer than its orbital period $T_{\mathrm{K}}$. Therefore, we take orbital averaging as

$$
\begin{aligned}
\langle\mathcal{F}\rangle & =\frac{1}{T_{\mathrm{K}}} \int_{0}^{T_{\mathrm{K}}} \mathcal{F} d t \\
& =\frac{1}{2 \pi} \int_{0}^{2 \pi} \mathcal{F} \frac{\left(1-e^{2}\right)^{3 / 2}}{(1+e \cos f)^{2}} d f
\end{aligned}
$$


The orbital average of Eqs. (19) and (20) and the mass loss rate leads to

$$
\begin{aligned}
\left\langle\frac{d a}{d t}\right\rangle & =\eta\left(\frac{1+e^{2}}{1-e^{2}} \bar{\psi}_{\mathrm{m}}+\frac{2 e}{1-e^{2}} \bar{\phi}_{\mathrm{m}}\right) \frac{\beta_{\mathrm{total}}}{1-\beta_{\mathrm{total}}} \frac{a}{m}-\frac{\beta_{\mathrm{PR}} G M_{\star}}{c} \frac{2+3 e^{2}}{a\left(1-e^{2}\right)^{3 / 2}}, \\
\left\langle\frac{d e}{d t}\right\rangle & =\eta\left(e \bar{\psi}_{\mathrm{m}}+\bar{\phi}_{\mathrm{m}}\right) \frac{\beta_{\text {total }}}{1-\beta_{\text {total }}} \frac{1}{m}-\frac{5 \beta_{\mathrm{PR}} G M_{\star}}{2 c} \frac{e}{a^{2}\left(1-e^{2}\right)^{1 / 2}}, \\
\left\langle\frac{d m}{d t}\right\rangle & =-\bar{\psi}_{\mathrm{m}},
\end{aligned}
$$

with

$$
\begin{aligned}
& \bar{\psi}_{\mathrm{m}}=-\frac{1}{2 \pi} \int_{0}^{2 \pi} \frac{d m}{d t} \frac{\left(1-e^{2}\right)^{3 / 2}}{(1+e \cos f)^{2}} d f \\
& \bar{\phi}_{\mathrm{m}}=-\frac{1}{2 \pi} \int_{0}^{2 \pi} \frac{d m}{d t} \cos f \frac{\left(1-e^{2}\right)^{3 / 2}}{(1+e \cos f)^{2}} d f
\end{aligned}
$$

The first terms on the r.h.s. of Eqs. (23) and (24) are orbital-averaged changes caused by an increase in the pressure force of Eq. (1) during sublimation, and, therefore, vanish without sublimation. The second terms are the changes by the P-R drag of Eq. (1). They are effective even if sublimation does not occur. Note that the first terms are positive for $\eta>0$ with which we are concerned and the second terms are always negative.

\subsection{ELLIPTIC EXPANSIONS}

When they drift radially inward by the P-R effect with small eccentricities and sublime in a certain zone, the orbital and mass evolution of dust particles is divided into three stages (Kobayashi et al. 2008). The three stages are depicted in Figs. 1 and 2, for the sake of simplicity, using spherical particles of radii $s$ and mass $m=4 \pi \rho s^{3} / 3$, where $\rho$ is the material density. Here we adopt physical values listed in Table 1 and $\gamma=0$, assuming 
that $P_{0}(T)$ is constant with $T$. In Stage I, the semimajor axis of a dust particle decreases mainly by the P-R drag while its mass gradually decreases as it approaches the central star. Reversely, in Stage II, the semimajor axis increases slightly while the mass loss due to sublimation is intensive. The duration in Stage II is determined by the mass-loss rate whose timescale is comparable to the P-R drift timescale in Stage II. In Stage III, the eccentricity pumps up to a value larger than 0.1 and the semimajor axis grows while the mass slightly decreases. The drift rate, which determines the number density, varies from stage to stage. In Stage I, the drift rate equals to that due to only the P-R drag, leading to a number density is in inverse proportion to distance. In Stage II, the drift rate becomes smaller than that in Stage I, because the inward drift is suppressed by an increase in $\beta$ due to mass loss by sublimation. This results in a pile-up of dust particles in Stage II. In Stage III, the radial drift turns to be outward and the orbit becomes highly eccentric $(e \gtrsim 0.1)$. As a result, the contribution of Stage III to the number density is negligible compared to those of Stages I and II. Therefore, the following analytic treatment for the number density will be made for Stages I and II.

Considering dust particles drifting radially inward by the P-R effect starting from small initial eccentricities $e_{0}$, say $e_{0}<0.1$, we expand Eqs. (23)-(25) in $e$ as

$$
\begin{aligned}
\left\langle\frac{d a}{d t}\right\rangle & =-\left.\eta \frac{\beta_{\text {total }}}{1-\beta_{\text {total }}} \frac{a}{m} \frac{d m}{d t}\right|_{r=a}-\frac{\beta_{\mathrm{PR}} G M_{\star}}{c} \frac{2}{a}+O\left(e^{2}\right) \\
\left\langle\frac{d e}{d t}\right\rangle & =-\left.\eta e\left(\frac{1}{4}-\frac{1}{2} \frac{d \ln P_{\mathrm{v}}}{d \ln T}\right) \frac{d \ln T}{d \ln a} \frac{\beta_{\mathrm{total}}}{1-\beta_{\mathrm{total}}} \frac{1}{m} \frac{d m}{d t}\right|_{r=a}-\frac{5 \beta_{\mathrm{PR}} G M_{\star}}{2 c} \frac{e}{a^{2}}+O\left(e^{2}\right), \\
\left\langle\frac{d m}{d t}\right\rangle & =\left.\frac{d m}{d t}\right|_{r=a}+O\left(e^{2}\right)
\end{aligned}
$$


where $d m /\left.d t\right|_{r=a}$ is $d m / d t$ at $r=a$. We can neglect the terms of the order $e^{2}$ and higher if $e \ll\left(d \ln P_{\mathrm{v}} / d \ln T\right)^{-1}|\partial \ln T / \partial \ln a|^{-1} \sim k T / \mu m_{\mathrm{u}} H$.

For a dust particles in Stage II, its eccentricity increases by sublimation. The terms of the order of $e^{2}$ and higher in Eqs. (28)-(30) are not negligible if

$$
e \simeq\left(d \ln P_{\mathrm{v}} / d \ln T\right)^{-1}|\partial \ln T / \partial \ln a|^{-1}
$$

Then, $|\dot{m}|$ around the perihelion is much larger than that around the aphelion. The semimajor axis and eccentricity of a particle increase around the time of its perihelion passage without any substantial variation of the perihelion distance because the active sublimation dominates the dynamics. On the other hand, around the time of its aphelion passage, the perihelion distance as well as the semimajor axis and eccentricity decrease by the P-R effect. In one orbital period, the perihelion distance decreases although the semimajor axis and eccentricity in total increase because of the active sublimation around the perihelion. Because sublimation becomes more active with decreasing perihelion distance, the semimajor axis rapidly increases, namely, the particle is blown out. We defined this stage as Stage III.

From the energy balance equation (14), we calculate the total derivative $d \ln T / d \ln a$ along the orbital and mass evolution of a dust particle by replacing $r$ by $a$ in Eq. (14). The result is given (see Appendix B) by

$$
\frac{d \ln T}{d \ln a}=\frac{1}{4+c_{T}}\left[\frac{a}{m} \frac{\dot{m}}{\dot{a}}\left(c_{\star}-c_{\mathrm{d}}\right)-2 \Lambda\right]
$$


where

$$
\Lambda=\frac{1}{2}\left(\frac{R_{\star}}{a}\right)^{2} \frac{1}{\sqrt{1-\left(R_{\star} / a\right)^{2}}\left[1-\sqrt{1-\left(R_{\star} / a\right)^{2}}\right]},
$$

and

$$
c_{\star}=\frac{d \ln \bar{C}_{\star}}{d \ln m}, \quad c_{\mathrm{d}}=\left(\frac{\partial \ln \bar{C}_{\mathrm{d}}}{\partial \ln m}\right)_{T}, \quad c_{T}=\left(\frac{\partial \ln \bar{C}_{\mathrm{d}}}{\partial \ln T}\right)_{m} .
$$

Note that $\Lambda \simeq 1$ for $r \gg R \star$. For large dust particles that behave like blackbodies, both cross-sections $\bar{C}_{\star}$ and $\bar{C}_{\mathrm{d}}$ reduce to the geometrical cross-section. Consequently, we see $c_{T}=0$ and $c_{\star}=c_{\mathrm{d}}$, and we get the well-known result that $d \ln T / d \ln a=-\Lambda / 2 \simeq-1 / 2$ for $a \gg R_{\star}$

\subsection{ANALYTICAL FORMULAE OF ORBITAL EVOLUTION}

\subsubsection{STAGE I}

In Stage I, sublimation is negligible because of low temperature. The changes of the semimajor axis and the eccentricity are given by

$$
\begin{aligned}
& \frac{d a}{d t}=-\frac{2 G M \beta_{\mathrm{PR}}}{c a} \\
& \frac{d e}{d t}=-\frac{5 G M e \beta_{\mathrm{PR}}}{2 c a^{2}}
\end{aligned}
$$

neglecting the sublimation terms in Eqs. (28) and (29). This leads to the well-known relation

$$
\frac{a}{a_{0}}=\left(\frac{e}{e_{0}}\right)^{4 / 5}
$$


first obtained by Wyatt and Whipple (1950).

Although the sublimation timescale of a dust particle is much longer than the drift timescale in Stage I, the mass of a dust particle decreases as it approaches the central star. The thickness $l$ of the dust-particle surface layer decreases with time as

$$
\frac{d l}{d t}=\sqrt{\frac{\mu m_{\mathrm{u}}}{2 \pi k T}} \frac{P_{\mathrm{v}}}{\rho} .
$$

This comes from Eq. (12) assuming uniform sublimation from the surface, where $-d m=\rho A d l$. The thickness $l$ lost by sublimation during inward radial drift from $r=a_{0}$ to $a$ is given by

$$
l=\left.\int \frac{d l}{d t}\right|_{r=a} d t=\int_{T\left(r=a_{0}\right)}^{T(r=a)} \frac{\dot{l}}{\dot{a}}\left(\frac{\partial T}{\partial a}\right)_{m}^{-1} d T .
$$

In Stage I, the sublimation rate is so small that we can approximate $\dot{a}$ in Eq. (39) by that due to the P-R drag given by Eq. (35). Hence, $l$ is expressed as

$$
l \simeq \int_{T\left(a_{0}\right)}^{T(a)} F(T) e^{-\mu m_{\mathrm{u}} H / k T} d T
$$

where

$$
F(T)=\frac{c a P_{0}(T)}{2 G M_{\star} \rho \beta_{\mathrm{PR}}(m)} \sqrt{\frac{\mu m_{\mathrm{u}}}{2 \pi k T}}\left(-\frac{\partial T}{\partial a}\right)_{m}^{-1} .
$$

Since $F(T)$ is a slowly varying function of $T$ compared to $e^{-\mu m_{\mathrm{u}} H / k T}$, Eq. (40) is integrated in a good accuracy to be:

$$
\begin{aligned}
l(a, m) & \simeq \frac{k T^{2}(a)}{\mu m_{\mathrm{u}} H} F(T(a)) e^{-\mu m_{\mathrm{u}} H / k T(a)} \\
& \simeq \frac{\left(4+c_{T}\right) c a^{2}}{4 G M_{\star} \beta_{\mathrm{PR}} \Lambda} \sqrt{\frac{\mu m_{\mathrm{u}}}{2 \pi k T}} \frac{P_{\mathrm{v}}}{\rho} \frac{k T^{2}}{\mu m_{\mathrm{u}} H}
\end{aligned}
$$


for $T(a)>T\left(a_{0}\right)$, where we use $\partial \ln T / \partial \ln a=-2 \Lambda /\left(4+c_{T}\right)$ in obtaining the last expression.

Once the sublimated thickness $l$ is known, the dust mass $m$ at $r=a$ is given by

$$
-\int_{m_{0}}^{m} \frac{d m^{\prime}}{A\left(m^{\prime}\right) \rho\left(m^{\prime}\right)}=l
$$

For a dust particle having the relation between $\rho A$ and mass expressed by $A \rho \propto m^{\xi}$ with $\xi$ being a constant, the mass at $r=a$ is given by

$$
m=m_{0}\left[1-\frac{\rho_{0} A_{0} l}{m_{0}}(1-\xi)\right]^{\frac{1}{1-\xi}}
$$

where $A_{0}$ is the initial surface area, $\rho_{0}$ is the initial density, and $l$ is given by Eq. (42). For $\rho_{0} A_{0} l / m_{0} \ll 1, m \simeq m_{0}-\rho_{0} A_{0} l$.

The relevant formula for a spherical particle is given in Appendix A.2.

\subsubsection{STAGE II}

In Stage II, the drift rate $\left|\dot{a}_{\text {sub }}\right|$ of the first term on the r.h.s. of Eq. (28) becomes comparable to $\left|\dot{a}_{\mathrm{PR}}\right|$ of the second term, resulting in $|\dot{a}|=\left|\dot{a}_{\text {sub }}+\dot{a}_{\mathrm{PR}}\right| \ll\left|\dot{a}_{\mathrm{PR}}\right|$. Small $|\dot{a}|$ leads to an accumulation of dust particles in the relevant region. For the dust particles to pile up, $\eta=-d \ln \beta_{\text {total }} / d \ln m$ must be positive, implying that the importance of pressure force increases with decreasing size. With the assumption that the temporal variation of $a$ is small, the drift rate $d a /\left.d t\right|_{\text {II }}$ in Stage II is calculated (see Appendix C) to be:

$$
\left.\frac{d a}{d t}\right|_{\mathrm{II}}=\frac{2 G M_{\star} \beta_{\mathrm{PR}}(m)\left[1-\beta_{\mathrm{total}}(m)\right]}{c a \eta \beta_{\mathrm{total}}(m) g_{\mathrm{m}}(T, m)}=-\dot{a}_{\mathrm{PR}}(m) \frac{1-\beta_{\mathrm{total}}(m)}{\eta \beta_{\mathrm{total}}(m) g_{\mathrm{m}}(T, m)}
$$


where

$$
\dot{a}_{\mathrm{PR}}(m)=-\frac{2 G M_{\star} \beta_{\mathrm{PR}}(m)}{c a}
$$

is the P-R drift rate of a dust particle of mass $m$ and

$$
g_{\mathrm{m}}(T, m)=\frac{\frac{2 \Lambda}{4+c_{T}}\left(\frac{d \ln P_{\mathrm{V}}}{d \ln T}-\frac{1}{2}\right)-2}{1+\frac{\eta}{1-\beta_{\text {total }}}-\eta_{\mathrm{PR}}-\zeta_{A}-\frac{d \ln \eta}{d \ln m}-\left(\frac{d \ln P_{\mathrm{V}}}{d \ln T}-\frac{1}{2}\right) \frac{c_{\star}-c_{\mathrm{d}}}{4+c_{T}}}
$$

with $\eta_{\mathrm{PR}} \equiv-d \ln \beta_{\mathrm{PR}} / d \ln m$ and $\zeta_{A}=d \ln A / d \ln m$. The drift rate given by Eq. (45) is a good approximation for $|\langle d a / d t\rangle| \ll|d a / d t|_{\mathrm{PR}}$ and $d^{2} a / d t^{2} \ll|\langle d a / d t\rangle||d a / d t|_{\mathrm{PR}} / a$. Since $\langle d a / d t\rangle \simeq 0\left(|\langle d a / d t\rangle| \ll \dot{a}_{\mathrm{PR}}\right)$ in Stage II, Eq. (45) is also expressed by

$$
\left.\frac{1}{a} \frac{d a}{d t}\right|_{\mathrm{II}}=-\frac{1}{g_{\mathrm{m}}(T, m)} \frac{1}{m} \frac{d m}{d t}
$$

with the use of Eq. (28) (see Appendix C). Equation (45) indicates that a substantial number of dust particles pile up, if

$$
\frac{\beta_{\text {total }}}{1-\beta_{\text {total }}} \eta g_{\mathrm{m}} \gg 1
$$

This is also the condition for Eq. (45) to hold as stated above.

For a large blackbody-like dust (i.e., $c_{\mathrm{d}}-c_{\star} \simeq 0$ ) with $\eta \simeq \eta_{\mathrm{PR}} \simeq 1-\zeta_{A}$, and $d \ln \eta / d \ln m \ll 1, g_{\mathrm{m}}(T, m)$ reduces to

$$
g_{\mathrm{m}}(T, m) \simeq \frac{(1-\beta) \Lambda}{2 \eta} \frac{\mu m_{\mathrm{u}} H}{k T}
$$

if $d \ln P_{\mathrm{v}} / d \ln T \simeq \mu m_{\mathrm{u}} H / k T \gg 1$ and $\beta_{\text {total }} \simeq \beta$. Thus, from Eqs. (49) and (50), a large number of large blackbody-like dust particles piles up, if

$$
\frac{2 k T}{\mu m_{\mathrm{u}} H} \ll \beta<1 .
$$


Since $\beta$ decreases with increasing dust mass (or size) in general, Eq. (51) sets an upper limit of the size of pile-up particles. The upper limit of the dust size is determined from the condition that $\beta=2 k T / \mu m_{\mathrm{u}} H \simeq 0.04$. For spherical compact particles of density $\rho=1 \mathrm{~g} \mathrm{~cm}^{-3}$, the upper limit is approximately $14 \mu \mathrm{m}$ for $L_{\star}=L_{\odot}$ and $M_{\star}=M_{\odot}$. For a star with a larger luminosity-to-mass ratio $L_{\star} / M_{\star}$, even larger particles may pile up.

For non-blackbody dust, $c_{\star}-c_{\mathrm{d}} \neq 0$ and the last term in the denominator of the r.h.s. of Eq. (47) can dominate the other terms. Then, $g_{\mathrm{m}}$ reduces to

$$
g_{\mathrm{m}}(T, m) \simeq \frac{2 \Lambda}{c_{\mathrm{d}}-c_{\star}}
$$

since $d \ln P_{\mathrm{v}} / d \ln T \gg 1$. This situation arises when a dust particle is non-blackbody and its size lies between the wavelength of the maximum stellar radiation spectrum and a typical wavelength of thermal radiation. In this case, the pile-up condition is given by $2 \eta \Lambda \beta_{\text {total }} /\left(1-\beta_{\text {total }}\right) \gg c_{\mathrm{d}}-c_{\star}$, but the degree of the pile-up is smaller than that for a large blackbody-like dust particle. The degree of the pile-up approaches that of the blackbody, when the dust size becomes larger than the dominant wavelength of thermal emission.

Equating the drift rate $\langle d a / d t\rangle$ in Eq. (28) with $d a /\left.d t\right|_{\text {II }}$ given by Eq. (45), we obtain a relation between $a$ and $m$ in Stage II as

$$
\eta \frac{\beta_{\text {total }}}{1-\beta_{\text {total }}} \frac{a}{m} A P_{\mathrm{v}} \sqrt{\frac{\mu m_{\mathrm{u}}}{2 \pi k T}}-\frac{2 G M_{\star} \beta_{\mathrm{PR}}}{c a}=\frac{2 G M_{\star} \beta_{\mathrm{PR}}\left(1-\beta_{\mathrm{total}}\right)}{c a \eta \beta_{\text {total }} g_{\mathrm{m}}(T, m)} .
$$

Here, quantities other than $a$ and $m$ are functions of $a$ and $m$. Note that the r.h.s is much smaller than each of the terms on the l.h.s. Equation (53) indicates that the drift rate $d a /\left.d t\right|_{\mathrm{II}}$ in Stage II is independent of the initial values of $a_{0}, e_{0}$, and $m_{0}$. 
Next we investigate the orbital evolution of eccentricity $e$ in Stage II. Eq. (29) may be written as

$$
\left.\frac{d e}{d t}\right|_{\mathrm{II}}=-e \eta \frac{\beta_{\mathrm{total}}}{1-\beta_{\mathrm{total}}} \frac{1}{m} \frac{d m}{d t}\left[\left(\frac{1}{4}-\frac{1}{2} \frac{d \ln P_{\mathrm{v}}}{d \ln T}\right) \frac{d \ln T}{d \ln a}-\frac{5}{4}\right]
$$

since $\langle d a / d t\rangle=0$ in Stage II. Using Eq. (48), $d \ln T / d \ln a$ given by Eq. (32) is expressed by

$$
\frac{d \ln T}{d \ln a}=-\frac{1}{4+c_{T}}\left[2 \Lambda+\left(c_{\star}-c_{\mathrm{d}}\right) g_{\mathrm{m}}(T, m)\right]
$$

Thus, we have

$$
\left.\frac{1}{e} \frac{d e}{d t}\right|_{\mathrm{II}}=-\kappa \eta \frac{\beta_{\text {total }}}{1-\beta_{\text {total }}} \frac{1}{m} \frac{d m}{d t}
$$

with

$$
\kappa=\frac{1}{4+c_{T}}\left(\frac{d \ln P_{\mathrm{v}}}{d \ln T}-\frac{1}{2}\right)\left[\Lambda+\frac{1}{2}\left(c_{\star}-c_{\mathrm{d}}\right) g_{\mathrm{m}}(T, m)\right]-\frac{5}{4}
$$

We may assume that $\kappa$ is constant during the evolution in Stage II, because dust particles keep a almost constant in Stage II. Recalling $\eta=-d \ln \beta_{\text {total }} / d \ln m$, Eq. (56) may be integrated to be:

$$
e=\left(\frac{1-\beta_{\text {total }}\left(m_{1}\right)}{1-\beta_{\text {total }}(m)}\right)^{\kappa} e_{1}
$$

where $e_{1}$ and $m_{1}$ are the eccentricity and mass of a dust particle just after entering Stage II.

Appendix A.3 gives relevant formulae for spherical particles.

Figures 1 and 2 compare the orbital and radius evolutions of spherical particles calculated by the analytic formulae with those by numerical integrations of Eqs. (23)-(25) for silicate (Fig. 1) and carbon (Fig. 2). We use the physical values listed in Table 1 and we approximate $B_{\star}(\lambda)$ by $B\left(\lambda, T_{\star}\right)$. Both Eqs. (37) and (44) in Stage I and Eqs. (53) and (58) 
in Stage II are confirmed to agree fairly well with the numerical integrations for $e \lesssim 10^{-1}$. For $e \gtrsim 10^{-1}$ (in Stage III), Eqs. (53) and (58) are no longer valid because terms on the order of $e^{2}$ cannot be neglected.

\subsection{ENHANCEMENT FACTOR}

We here derive the enhancement factors of number density and optical depth of dust particles at the sublimation zone from the analytic solutions to the orbital and mass evolution in Stage I and Stage II given in $\S$ 3.3. Note that the orbital inclinations of dust particles are kept constant throughout the evolution under sublimation and the P-R effect. Assuming that the initial eccentricity is small enough, the eccentricities in Stage I and Stage II remain rather small. In Stage I and Stage II, therefore, we can replace a semimajor axis $a$ by a heliocentric distance $r$. In Stage III dust particles have much larger eccentricities but their contribution to the total number density is negligible. We consider two adjacent evolution lines starting from two infinitesimally different points $\left(r_{0}, m_{0}\right)$ and $\left(r_{0}, m_{0}+d m_{0}\right)$ in the $r-m$ plane, which pass through $(r, m)$ and $(r, m+d m)$, respectively. The number of dust particles crossing the line between $(r, m)$ and $(r, m+d m)$ per unit time is constant along the "tube" enclosed by the two adjacent evolution lines. The number conservation of dust particles in a steady state can be written by $r^{2} n(m, r) \dot{a}(r) d m=r_{0}^{2} n\left(m_{0}, r_{0}\right) \dot{a}\left(r_{0}\right) d m_{0}$, where $\dot{a}(r)$ is the drift velocity of the dust particles at $r$, and $n(m, r) d m$ is the number density of dust particles at $r$. 
Considering a constant flux of dust particles of masses ranging from $m_{0 \min }$ to $m_{0 \max }$ due to the P-R drift from $r_{0}$, the evolution lines starting from $r_{0}$ and different masses do not overlap in Stage I but converge in a evolution line determined by Eq. (53) in Stage II. However, only the dust particles of initial masses ranging from $m_{\text {init,min }}(r)$ to $m_{\text {init,max }}(r)$ can reach distance $r$ where their masses are distributed from $m_{\min }$ to $m_{\max }$. Using the number conservation equation, the number density of dust particles at distance $r$ is

$$
N(r)=\int_{m_{\min }}^{m_{\max }} n(m, r) d m=\left(\frac{r_{0}}{r}\right)^{2} \int_{m_{\text {init, } \min }(r)}^{m_{\text {init,max }}(r)} \frac{\left|\dot{a}_{0}\right|}{|\dot{a}|} n\left(m_{0}, r_{0}\right) d m_{0} \text {, }
$$

where $\dot{a}_{0}=\dot{a}\left(r_{0}\right)$.

All the dust particles are initially in Stage I. Their initial drift velocity and initial number density at $r_{0}$ are, respectively, given by

$$
\begin{aligned}
\dot{a}_{0} & =-\frac{2 G M_{\star} \beta_{\mathrm{PR}}}{c r_{0}}, \\
N_{0} & =\int_{m_{0 \min }}^{m_{0 \max }} n\left(m_{0}, r_{0}\right) d m_{0},
\end{aligned}
$$

where $\beta_{\text {total }}\left(m_{0 \text { min }}\right)<0.5$. With an assumption of $m_{\text {init,min }}(r)=m_{0 \text { min }}$ and $m_{\text {init,max }}(r)=$ $m_{0 \max }$, the steady-state number density in Stage I is obtained from substituting Eqs. (35) and (60) to Eq. (59) as

$$
N_{\mathrm{I}}(r)=\frac{r_{0}}{r} \int_{m_{0 \min }}^{m_{0 \max }} \frac{\beta_{\mathrm{PR}}\left(m_{0}\right)}{\beta_{\mathrm{PR}}\left(m_{\mathrm{I}}\right)} n\left(m_{0}, r_{0}\right) d m_{0},
$$

where $m_{\mathrm{I}}\left(m_{0}, r\right)$ is determined by Eq. (43).

In Stage II, the drift velocity $\dot{a}$ given by Eq. (45) is much smaller than that in Stage I $\left(\dot{a}_{\mathrm{PR}}\right)$, if condition (49) is satisfied. The small drift velocity in Stage II leads to the 
accumulation of dust particles in the sublimation zone. Note that the Stage II drift velocity given by Eq. (45) does not depend on the initial semimajor axis $a_{0}$ nor mass $m_{0}$, which can be understood from the fact that all the evolution paths starting from the Stage I region in the $a-m$ plane are confluent to a single path in Stage II (see Eq. 53). From substituting Eqs. (45) and (60) to Eq. (59), the steady-state number density of dust particles in Stage II is thus given by

$$
N_{\mathrm{II}}(r)=\frac{r_{0}}{r} \frac{\eta\left(m_{\mathrm{II}}\right) g_{\mathrm{m}}\left(T, m_{\mathrm{II}}\right) \beta_{\mathrm{total}}\left(m_{\mathrm{II}}\right)}{\beta_{\mathrm{PR}}\left(m_{\mathrm{II}}\right)\left[1-\beta_{\text {total }}\left(m_{\mathrm{II}}\right)\right]} \int_{m_{\text {init,min }}(r)}^{m_{\text {init } \max }(r)} \beta_{\mathrm{PR}}\left(m_{0}\right) n\left(m_{0}, r_{0}\right) d m_{0},
$$

where $m_{\mathrm{II}}(r)$ is the dust particles' mass at $r$ in Stage II given by Eq. (53).

We also calculate the effective optical depth $\tau_{\text {eff }}(r)$ for dust particles with the orientation-averaged geometrical cross section $S$ in a geometrically thin disk so that one could compare with observations of debris disks. In steady state, the number conservation integrated vertically through the disk is given by $r n_{\mathrm{s}}(m, r) \dot{a} d m=r_{0} n_{\mathrm{s}}\left(m_{0}, r_{0}\right) \dot{a}_{0} d m_{0}$, where $n_{\mathrm{s}}$ is $n$ integrated vertically in the disk. From the integrated number-density conservation equation, the effective optical depth at $r$ is given by

$$
\tau_{\text {eff }}(r)=\int_{m_{\min }}^{m_{\max }} S(m) n_{\mathrm{s}}(m, r) d m=\frac{r_{0}}{r} \int_{m_{\mathrm{init}, \min }(r)}^{m_{\mathrm{init}, \max }(r)} \frac{\dot{a}_{0}}{\dot{a}} n_{\mathrm{s}}\left(m_{0}, r_{0}\right) d m_{0} .
$$

Here the initial effective optical depth at $r_{0}$ is calculated by

$$
\tau_{\mathrm{eff} 0}=\int_{m_{0 \min }}^{m_{0 \max }} S\left(m_{0}\right) n_{\mathrm{s}}\left(m_{0}, r_{0}\right) d m_{0}
$$

In Stage I, the steady-state effective optical depth is obtained from substituting Eqs. (35) 
and (60) to Eq. (64),

$$
\tau_{\mathrm{eff}, \mathrm{I}}(r)=\int_{m_{0 \min }}^{m_{0 \max }} \frac{\beta_{\mathrm{PR}}\left(m_{0}\right)}{\beta_{\mathrm{PR}}\left(m_{\mathrm{I}}\right)} S\left(m_{\mathrm{I}}\right) n_{\mathrm{s}}\left(m_{0}, r_{0}\right) d m_{0}
$$

because $m_{\text {init,min }}(r)=m_{0 \min }$ and $m_{\text {init,max }}=m_{0 \max }$.

In Stage II, from substituting Eqs. (45) and (60) to Eq. (64), the steady-state effective optical depth $\tau_{\text {eff,II }}(r)$ is given by

$$
\tau_{\text {eff }, \mathrm{II}}(r)=\frac{\eta\left(m_{\mathrm{II}}\right) g_{\mathrm{m}}\left(T, m_{\mathrm{II}}\right) \beta_{\mathrm{total}}\left(m_{\mathrm{II}}\right)}{\beta_{\mathrm{PR}}\left(m_{\mathrm{II}}\right)\left[1-\beta_{\mathrm{total}}\left(m_{\mathrm{II}}\right)\right]} S\left(m_{\mathrm{II}}\right) \int_{m_{\text {init,min }}(r)}^{m_{\text {init,max }}(r)} \beta_{\mathrm{PR}}\left(m_{0}\right) n_{\mathrm{s}}\left(m_{0}, r_{0}\right) d m_{0}
$$

In Stage II, dust particles pass the region where they did in Stage I as shown in Figs. 1 and 2. In the sublimation zone, therefore, the number density is practically given by $N_{\text {II }}+N_{\text {I }}$ and the effective optical depth is $\tau_{\text {eff,II }}+\tau_{\text {eff,I }}$. We neglect the mass loss of a dust particle due to sublimation in Stage I before starting Stage II, because it is much smaller than the initial mass $m_{0}$ for $\beta_{\text {total }}\left(m_{0}\right) \gtrsim 0.1$. Then, we can set $r N_{\mathrm{I}}=r_{0} N_{0}$ and $\tau_{\text {eff,I }}=\tau_{\text {effo } 0}$. We consider the smallest dust particles initially contribute most to the number density and the optical depth, and we pay attention to the peak of $r N_{\text {II }}$ and $\tau_{\text {eff,II }}$, at which we derive $N_{\text {II }}$ and $\tau_{\text {eff,II }}$ by setting $m_{\text {init,min }}=m_{\text {II }}=m_{0 \min }$ in Eqs. (63) and (67). Assuming that $n\left(a_{0}, m_{0}\right) \propto m_{0}^{-b}, \beta_{\text {total }}(m) \propto m^{-\eta\left(m_{0 \min }\right)}$, and $S \propto m^{\zeta_{S}}$, the number-density enhancement factor $f_{N}$ and the effective-optical-depth enhancement factor $f_{\tau_{\text {eff }}}$ at the peak are given by

$$
\begin{aligned}
f_{N} & =\frac{N_{\mathrm{II}}+N_{\mathrm{I}}}{N_{0}} \frac{r}{r_{0}} \\
& \simeq \frac{\eta\left(m_{0 \min }\right) \beta_{\text {total }}\left(m_{0 \mathrm{~min}}\right)}{1-\beta_{\text {total }}\left(m_{0 \min }\right)} g_{\mathrm{m}}\left(T_{\mathrm{sub}}, m_{0 \min }\right) h_{1}\left(y_{1}, y_{2}\right)+1, \\
f_{\tau_{\mathrm{eff}}} & =\frac{\tau_{\text {eff } \mathrm{II}}+\tau_{\text {eff }, \mathrm{I}}}{\tau_{\text {effo }}}
\end{aligned}
$$




$$
\simeq \frac{\eta\left(m_{0 \min }\right) \beta_{\text {total }}\left(m_{0 \min }\right)}{1-\beta_{\text {total }}\left(m_{0 \min }\right)} g_{\mathrm{m}}\left(T_{\mathrm{sub}}, m_{0 \min }\right) h_{2}\left(y_{1}, y_{2}\right)+1
$$

where

$$
\begin{aligned}
& h_{1}\left(y_{1}, y_{2}\right)=\frac{\int_{1}^{y_{1}} \tilde{y}^{-b-\eta\left(m_{0 \min }\right)} d \tilde{y}}{\int_{1}^{y_{2}} \tilde{y}^{-b} d \tilde{y}}, \\
& h_{2}\left(y_{1}, y_{2}\right)=\frac{\int_{1}^{y_{1}} \tilde{y}^{-b-\eta\left(m_{0 \min }\right)} d \tilde{y}}{\int_{1}^{y_{2}} \tilde{y}^{-b+\zeta_{S}} d \tilde{y}},
\end{aligned}
$$

$y_{1}=m_{\text {init,max }} / m_{0 \min }$ and $y_{2}=m_{0 \max } / m_{0 \min }$. For small eccentricity, we set $y_{1}=y_{2}=$ $m_{0 \max } / m_{0 \min }$ for $m_{\text {init,max }}=m_{0 \max }$. The temperature $T_{\text {sub }}$ is determined by Eqs. (14) and (53) for the mass $m_{0 \mathrm{~min}}$. We derive the enhancement factors for spherical particles in Appendix A.4.

We show the scaled number density $\left(r N / r_{0} N_{0}\right)$ for spherical particles in Figs. 3 and 4 numerically calculated with the method described in Kobayashi et al. (2008). If we set the initial parameters of $a_{0}=6 R_{\odot}, e_{0}=10^{-4}, b=11 / 6$, and $s_{0}=1-5 \mu \mathrm{m}\left(m_{0 \mathrm{~min}}=9.9 \times 10^{-12} \mathrm{~g}\right.$ and $m_{0 \max }=1.2 \times 10^{-9} \mathrm{~g}$ for silicate and $m_{0 \min }=8.1 \times 10^{-12} \mathrm{~g}$ and $m_{0 \max }=1.0 \times 10^{-9} \mathrm{~g}$ for carbon), the number-density enhancement factor estimated from Eq. (68) is 3.4 at $4.3 R_{\odot}$ for silicate and 10 at $3.6 R_{\odot}$ for carbon. The sublimation distance is determined by Eqs. (14) and (53) for the mass $m_{0 \mathrm{~min}}$. These values are in agreement with the numerical results (see Figs. 5 and 6) and previous studies (e.g., Mukai and Yamamoto 1979).

As a demonstration of these formulae, we calculate $f_{N}, f_{\tau_{\text {eff }}}$, and $a_{\text {sub }}$ from Eqs. (68), (69), (14), and (53) for spherical particles of silicate and carbon in extrasolar systems, where $a_{\text {sub }}$ is the distance at the peak of the number density divided by $1 / r, r N$, and the effective optical depth $\tau_{\text {eff }}$ (see Figs. 5 and 6) and is derived from Eq. (53) for $m=m_{0 \min }$. 
We set the power-law index of the mass distribution of drifting dust as $b=11 / 6$. We chose the smallest initial mass $m_{0 \min }$ as the mass corresponding to $\beta_{\text {total }}=0.5$, because a dust particle with $\beta_{\text {total }}>0.5$ has an unbound orbit just after the dust particle is produced. The largest initial mass $m_{0 \max }$ is chosen as the mass corresponding to $\beta_{\text {total }}=0.1$, because a dust particle with a smaller $\beta_{\text {total }}$ value does not effectively drift into the sublimation zone owing to its slow radial drift. We set $L_{\star} \propto M_{\star}^{3.5}$ and $T_{\star} \propto L_{\star}^{0.12}$ for the stellar parameters (Allen 1976).

We confirm that the enhancement factors and the sublimation distance agree with those of the numerical calculations for solar luminosity. The distance $a_{\text {sub }}$ significantly depends on stellar luminosity. The temperature $T_{\text {sub }}$ at the peak of $r N$ and $\tau_{\text {eff }}$ is almost independent of the luminosity, which is about $1200 \mathrm{~K}$ for silicate and $2000 \mathrm{~K}$ for carbon. The peak distance $a_{\text {sub }}$ is determined by Eq. (53) for the smallest dust at $T_{\text {sub }}$ because the dust contributes most to $N$ and $\tau_{\text {eff }}$. For high luminosity, the minimum size of dust particles is much larger than the wavelength $\lambda_{\text {sub }}$ at the maximum thermal radiation because small dust cannot resist against high radiation pressure. The distance $a_{\text {sub }}$ for silicate is larger than that for carbon in geometrical optics because $T_{\text {sub }}$ for silicate is colder than that for carbon. For low luminosity, the peak distance depends on the optical properties because the smallest dust becomes as small as $\lambda_{\text {sub }}$. The distance for silicate becomes small relative to that for carbon because silicate dust is less absorbing than carbon one. The distance $a_{\text {sub }}$ for both silicate and carbon are comparable for $L_{\star}=L_{\odot}$, accidentally. 
For high luminosity, $m_{0 \mathrm{~min}}$ becomes large because of strong radiation pressure. The enhancement factors strongly depend on $(\partial \ln T / \partial \ln m)_{a} \simeq\left(c_{\star}-c_{\mathrm{d}}\right) /\left(4+c_{T}\right)$ for $m_{0 \text { min }}$ (see $g_{\mathrm{m}}$ in Eqs. 68 and 69). In Figs. 5 and 6, the enhancement factors have peaks where the values of $(\partial \ln T / \partial \ln m)_{a}$ become minimum.

\section{APPLICATION OF FORMULAE}

We have derived an analytical formula for the number-density enhancement factor of a dust ring formed by sublimation of dust particles drifting inward by the P-R drag (see Eq. 68). Such a dust ring is ubiquitous, because the enhancement factor is always larger than unity in any stellar luminosities as shown in Figs. 5 and 6 . The enhancement factor is mainly determined by the flux of the smallest dust particles of mass $m_{0 \text { min }}$ drifting into the sublimation zone. The analytical formula of Eq. (68) can be applied to any given systems as long as drag-dominated disks are concerned. The enhancement factor strongly depends on the temperature $T_{\text {sub }}$ through $g_{\mathrm{m}}\left(T_{\mathrm{sub}}, m_{0 \mathrm{~min}}\right)$ given by Eq. $(47)$, where $T_{\text {sub }}$ is determined by Eqs. (14) and (53) using $m_{0 \text { min }}$ corresponding to $\beta_{\text {total }}=0.5$.

One may expect that a dust ring of subliming particles forms in typical debris disks around stars such as $\beta$ Pictoris and Vega. Contrary to our basic assumption of drag-dominated disks, these prominent debris disks are collision-dominated. Their optical depths are so high that collision timescales are much shorter than P-R timescales. Nevertheless, these debris disks will eventually evolve in drag-dominated disks by collisional 
clean-up. Therefore, it is worth calculating enhancement factors of number densities at the outer edges of dust sublimation zones in the hypothetical drag-dominated disks around these stars. Table 2 gives the enhancement factor $f_{N}$ of a dust ring as well as the sublimation temperature $T_{\text {sub }}$ for icy, silicate, or carbon dust particles estimated with stellar parameters of the sun, $\beta$ Pictoris, and Vega. Also listed is the ring radius $a_{\text {sub }}$, which is simultaneously obtained during the determination of $T_{\text {sub }}$. We predict that dust rings due to sublimation will be discovered around typical drag-dominated disks characterized by a vertical optical depth smaller than $10^{-5}$, on account of progress in observation of such faint and evolved disks.

\section{DISCUSSION}

We have presented an analytical model for the dynamics of subliming dust particles that radially drift toward a central star by the P-R drag. The analytical formulae given in this paper describe the orbital and mass evolution of the dust particles including their radial drift rates. Using the drift rates, we have also derived analytical formulae for the number density and effective optical depth along with their enhancement factors at the outer edge of the sublimation zone. These formulae are in agreement with numerical results for spherical dust particles of silicate, carbon, or dirty ice (cf. Kobayashi et al. 2008). They would be applicable not only to spherical particles but also to fluffy dust aggregates. Previous numerical studies show that the enhancement factor for fluffy dust aggregates is smaller 
than that for spherical particles (Kimura et al. 1997; Mann et al. 2000). Equations (68) and (69) indicate that the enhancement factors are in proportion to $\eta=-d \ln \beta / d \ln m$. A dust particle with a fractal structure has $d \ln \beta / d \ln m \sim-(D-2) / D$ unless $D<2$, where $D$ is a fractal dimension of the dust particle (Mukai et al. 1992; Köhler et al. 2007). As a result, the enhancement factors decrease as $D$ becomes close to 2 . This is consistent with the previous studies, in which fluffier particles having smaller $D$ are less accumulated at the outer edge of the sublimation zone. This demonstrates the applicability of our analytical model to dust particles of arbitrary shapes and materials.

When eccentricities of dust particles drifting into the sublimation zone are smaller than $10^{-4}$, the enhancement factors are constant with the eccentricities but smaller for larger eccentricities (Kobayashi et al. 2008). Eccentricities of dust particles grow according to Eq. (58) in Stage II starting from $e_{1}$, which is determined by a set of $a_{0}$ and $e_{0}$ according to the orbital evolution due to the P-R effect (see Eq. 37). When eccentricities become as large as 0.1, the dust particles are blown out in Stage III. For large $e_{1}$, dust particles begin to be blown out even after small mass loss due to sublimation. Therefore, the pile-up hardly happens for $e_{1} \gtrsim 0.1$ because dust particles spend too short a time in Stage II. Initially large particles are blown out before they reach the peak values of the number density and the optical depth. The number density and optical depth become small because of the low value of $m_{\text {init,max }}$ in Eqs. (63) and (67). We conclude that the enhancement factors would be valid as long as $e_{1} \lesssim 0.1$ if the dependence of $m_{\text {init,max }}$ on $e_{1}$ according to Eq. (58) is taken into account. 
We have indicated that Eq. (49) gives the condition for dust particles to form a ring around a central star. For $\eta \beta_{\text {total }} g_{\mathrm{m}}(T, m) \gg 1-\beta_{\text {total }}$, the drift rate becomes small because of the equilibrium between the inward drift due to the P-R effect and the outward one due to sublimation, resulting in a dust pile-up at the sublimation zone. The enhancement factor becomes larger than $(2 b-1) / b$, where $b=-d \ln n_{\mathrm{s}, 0} / d \ln m_{0}$. On the other hand, for $\eta \beta_{\text {total }} g_{\mathrm{m}}(T, m) \ll 1-\beta_{\text {total }}$, the drift rate does not stay small because the temperature strongly increases during sublimation. As a result, the drift rate due to sublimation becomes much larger than that due to the P-R effect, so that dust does not pile up at the sublimation zone. Considering sublimation of dirty ice in the Solar System, spherical icy dust particles with $s \lesssim 2 \mu \mathrm{m}$ radially drifting from an outer region form a ring at 20-40 AU from the Sun. However, large dust particles of dirty ice do not form a ring because of $\eta \beta_{\text {total }} g_{\mathrm{m}}(T, m) \lesssim 1-\beta_{\text {total }}$. We expect that a dust ring of small icy dust particles will be detected by future interplanetary missions with a dust detector whose mass threshold is well below $2 \mu \mathrm{m}$.

We thank anonymous reviewers for their helpful comments. This research was supported by MEXT Japan, Grant-in-Aid for Scientific Research on Priority Areas, "Development of Extra-solar Planetary Science" (16077203) and "Astrophysical Observations of New Phases of the Interstellar Gas at Sub-mm and THz Regions" (18026001) and by JSPS, Grant-in-Aid for Young Scientists (B) (18740103) and Grant-in-Aid (C) (19540239). 


\section{A. CASE OF A SPHERICAL PARTICLE}

We consider a spherical particle with its radius $s$. We define the efficiency $\bar{Q}_{\mathrm{d}}=\bar{C}_{\mathrm{d}} / \pi s^{2}$ and $\bar{Q}_{\star}=\bar{C}_{\star} / \pi s^{2}$.

The sublimation rate is given by

$$
\frac{d s}{d t}=-\sqrt{\frac{\mu m_{\mathrm{u}}}{2 \pi k T}} \frac{P_{\mathrm{v}}}{\rho},
$$

because $A=4 \pi s^{2}$.

\section{A.1. ORBITAL-AVERAGED EVOLUTION RATES}

The orbital averaged evolution rates are given by

$$
\begin{aligned}
\left\langle\frac{d a}{d t}\right\rangle & =\alpha\left(\frac{1+e^{2}}{1-e^{2}} \bar{\psi}_{\mathrm{s}}+\frac{2 e}{1-e^{2}} \bar{\phi}_{\mathrm{s}}\right) \frac{\beta_{\text {total }}}{1-\beta_{\text {total }}} \frac{a}{s}-\frac{\beta_{\mathrm{PR}} G M_{\star}}{c} \frac{2+3 e^{2}}{a\left(1-e^{2}\right)^{3 / 2}}, \\
\left\langle\frac{d e}{d t}\right\rangle & =\alpha\left(e \bar{\psi}_{\mathrm{s}}+\bar{\phi}_{\mathrm{s}}\right) \frac{\beta_{\text {total }}}{1-\beta_{\text {total }}} \frac{1}{s}-\frac{5 \beta_{\mathrm{PR}} G M_{\star}}{2 c} \frac{e}{a^{2}\left(1-e^{2}\right)^{1 / 2}}, \\
\left\langle\frac{d s}{d t}\right\rangle & =-\bar{\psi}_{\mathrm{s}},
\end{aligned}
$$

with

$$
\begin{aligned}
& \bar{\psi}_{\mathrm{s}}=-\frac{1}{2 \pi} \int_{0}^{2 \pi} \frac{d s}{d t} \frac{\left(1-e^{2}\right)^{3 / 2}}{(1+e \cos f)^{2}} d f \\
& \bar{\phi}_{\mathrm{s}}=-\frac{1}{2 \pi} \int_{0}^{2 \pi} \frac{d s}{d t} \cos f \frac{\left(1-e^{2}\right)^{3 / 2}}{(1+e \cos f)^{2}} d f
\end{aligned}
$$

where $\alpha=-d \ln \beta_{\text {total }} / d \ln s$. 


\section{A.2. STAGE I}

In Stage I, the relation of $a$ and $e$ is given by Eq. (37). From Eq. (44), the relation of $a$ and $s$ is given by

$$
s=s_{0}-\sqrt{\frac{\mu m_{\mathrm{u}}}{2 \pi k T}} \frac{\left(4+c_{T}\right) c a^{2}}{4 \Lambda G M_{\star} \rho \beta_{\mathrm{PR}}(s)} \frac{k T^{2}}{\mu m_{\mathrm{u}} H} P_{\mathrm{v}}(T),
$$

where the temperature is a function of $a$ and $s_{0}$, namely, $T\left(a, s_{0}\right)$.

\section{A.3. STAGE II}

In Stage II, from Eq. (53), the relation between $a$ and $s$ is given by

$$
\alpha \frac{\beta_{\text {total }}}{1-\beta_{\text {total }}} \frac{a}{s} \sqrt{\frac{\mu m_{\mathrm{u}}}{2 \pi k T}} \frac{P_{\mathrm{v}}}{\rho}-\frac{\beta_{\mathrm{PR}} G M_{\star}}{c} \frac{2}{a}=\frac{2 G M_{\star} \beta_{\mathrm{PR}}\left(1-\beta_{\text {total }}\right)}{c a \alpha \beta_{\text {total }} g_{\mathrm{s}}(T, s)},
$$

with

$$
g_{\mathrm{s}}(T, s)=\frac{\frac{2 \Lambda}{4+q_{T}}\left(\frac{d \ln P_{\mathrm{v}}}{d \ln T}-\frac{1}{2}\right)-2}{1+\alpha-\alpha_{\mathrm{PR}}+\alpha \frac{\beta_{\text {total }}}{1-\beta_{\text {total }}}-\frac{d \ln \alpha}{d \ln s}-\left(\frac{d \ln P_{\mathrm{v}}}{d \ln T}-\frac{1}{2}\right) \frac{q_{\star}-q_{\mathrm{d}}}{4+q_{T}}},
$$

where $q_{\star}=d \ln \bar{Q}_{\star} / d \ln s, q_{\mathrm{d}}=\partial \ln \bar{Q}_{\mathrm{d}} / \partial \ln s, q_{T}=\partial \ln \bar{Q}_{\mathrm{d}} / \partial \ln T$, and $\alpha_{\mathrm{PR}}=d \ln \beta_{\mathrm{PR}} / d \ln s$.

For a blackbody dust particle $\left(q_{\star}=q_{\mathrm{d}}=0\right)$, the function of $g_{\mathrm{s}}(T, s)$ is reduced to

$$
g_{\mathrm{s}}(T, s)=\left(1-\beta_{\text {total }}\right)\left[\frac{2 \Lambda}{4+q_{T}}\left(\frac{d \ln P_{\mathrm{v}}}{d \ln T}-\frac{1}{2}\right)-2\right]
$$

Furthermore, when $\left|q_{\star}-q_{\mathrm{d}}\right| \gg\left(4+q_{T}\right)\left(d \ln P_{\mathrm{v}} / d \ln T\right)^{-1}$, the $g_{\mathrm{s}}$ function is approximately given by

$$
g_{\mathrm{s}}(T, s) \simeq 2 /\left(q_{\mathrm{d}}-q_{\star}\right)
$$

because of $d \ln P_{\mathrm{v}} / d \ln T \gg 1$. 
From Eq. (58), the relation between $s$ and $e$ is given by

$$
e=\left(\frac{1-\beta_{\text {total }}\left(s_{1}\right)}{1-\beta_{\text {total }}(s)}\right)^{\kappa_{\mathrm{s}}} e_{1}
$$

where

$$
\kappa_{\mathrm{s}}=\left(\frac{d \ln P_{\mathrm{v}}}{d \ln T}-\frac{1}{2}\right) \frac{1}{4+q_{T}}\left[\Lambda+\frac{q_{\star}-q_{\mathrm{d}}}{2} g_{\mathrm{s}}(T, s)\right]-\frac{5}{4}
$$

$e_{1}$ and $s_{1}$ are eccentricity and radius of a dust particle just after entering in Stage II

\section{A.4. ENHANCEMENT FACTOR}

From Eqs. (68) and (69), if we assume $n_{0} \propto s^{-p}$ and $\beta_{\text {total }}\left(s_{0 \min }\right) \propto s^{-\alpha\left(s_{0 \min }\right)}$, the enhancement factors $f_{N}$ and $f_{\tau_{\text {eff }}}$ are given by

$$
\begin{aligned}
f_{N} & \simeq \frac{\alpha\left(s_{0 \min }\right) \beta_{\text {total }}\left(s_{0 \text { min }}\right)}{\left[1-\beta_{\text {total }}\left(s_{0 \text { min }}\right)\right]} g_{\mathrm{s}}\left(T_{\mathrm{sub}}, s_{0 \min }\right) h_{\mathrm{s} 1}\left(z_{1}, z_{2}\right)+1 \\
f_{\tau_{\mathrm{eff}}} & \simeq \frac{\alpha\left(s_{0 \text { min }}\right) \beta_{\text {total }}\left(s_{0 \text { min }}\right)}{\left[1-\beta_{\text {total }}\left(s_{0 \text { min }}\right)\right]} g_{\mathrm{s}}\left(T_{\mathrm{sub}}, s_{0 \min }\right) h_{\mathrm{s} 2}\left(z_{1}, z_{2}\right)+1
\end{aligned}
$$

with

$$
\begin{aligned}
& h_{\mathrm{s} 1}\left(z_{1}, z_{2}\right)=\frac{\int_{1}^{z_{1}} \tilde{z}^{-p-\alpha\left(s_{0 \min }\right)} d \tilde{z}}{\int_{1}^{z_{2}} \tilde{z}^{-p} d \tilde{z}} \\
& h_{\mathrm{s} 2}\left(z_{1}, z_{2}\right)=\frac{\int_{1}^{z_{1}} \tilde{z}^{-p-\alpha\left(s_{0 \min }\right)} d \tilde{z}}{\int_{1}^{y_{2}} \tilde{y}^{-p+2} d \tilde{y}}
\end{aligned}
$$

where $s_{0 \max }$ is the maximum initial radius, $s_{0 \min }$ is the minimum one, and $z_{1}=y_{1}^{1 / 3}$ and $z_{2}=s_{0 \max } / s_{0 \min }=y_{2}^{1 / 3}$. For low $e_{0} \lesssim 10^{-4}, z_{1} \simeq z_{2}$ (Kobayashi et al. 2008). 


\section{B. CALCULATION OF $d \ln T / d \ln a$}

Replacing $r$ by $a$ in Eq. (14) and indicating the variables of each function explicitly, we obtain

$$
\begin{aligned}
\ln \left[1-\sqrt{1-\left(\frac{R_{\star}}{a}\right)^{2}}\right]+\ln \bar{C}_{\star}(m(a)) & =4 \ln T(a, m(a)) \\
& +\ln \bar{C}_{\mathrm{d}}(T(a, m(a)), m(a))+\mathrm{const}
\end{aligned}
$$

Differentiation of each term with respect to $\ln a$ yields

$$
\begin{aligned}
\frac{d}{d \ln a} \ln \left[1-\sqrt{1-\left(\frac{R_{\star}}{a}\right)^{2}}\right] & =-2 \Lambda, \\
\frac{d}{d \ln a} \ln \bar{C}_{\star}(m(a)) & =\frac{d \ln \bar{C}_{\star}(m)}{d \ln m} \frac{d \ln m}{d \ln a}=c_{\star} \frac{d \ln m}{d \ln a},
\end{aligned}
$$

where

$$
c_{\star}=\frac{d \ln \bar{C}_{\star}(m)}{d \ln m},
$$

and $\Lambda$ is defined by Eq. (33). The derivative of $\bar{C}_{\mathrm{d}}(T, m)$ is calculated to be:

$$
\begin{aligned}
\frac{d}{d \ln a} \ln \bar{C}_{\mathrm{d}}(T(a, m), m) & =\left(\frac{\partial \ln \bar{C}_{\mathrm{d}}}{\partial \ln T}\right)_{m} \frac{d \ln T}{d \ln a}+\left(\frac{\partial \ln \bar{C}_{\mathrm{d}}}{\partial \ln m}\right)_{T} \frac{d \ln m}{d \ln a} \\
& =c_{T} \frac{d \ln T}{d \ln a}+c_{\mathrm{d}} \frac{d \ln m}{d \ln a}
\end{aligned}
$$

where

$$
c_{T}=\left(\frac{\partial \ln \bar{C}_{\mathrm{d}}}{\partial \ln T}\right)_{m}, \quad c_{\mathrm{d}}=\left(\frac{\partial \ln \bar{C}_{\mathrm{d}}}{\partial \ln m}\right)_{T} .
$$

Note that $d \ln T / d \ln a$ is a total derivative, namely,

$$
\frac{d \ln T(a, m)}{d \ln a}=\left(\frac{\partial \ln T}{\partial \ln a}\right)_{m}+\left(\frac{\partial \ln T}{\partial \ln m}\right)_{a} \frac{d \ln m}{d \ln a}
$$


Substituting Eqs. (B2) to (B6) into Eq. (B1), we obtain

$$
\frac{d \ln T}{d \ln a}=\frac{1}{4+c_{T}}\left[\frac{d \ln m}{d \ln a}\left(c_{\star}-c_{\mathrm{d}}\right)-2 \Lambda\right] .
$$

This is identical to Eq. (32).

For comparison, let us calculate partial derivatives of $\ln T$. Setting $m=$ const in Eq. (B8), we obtain

$$
\left(\frac{\partial \ln T}{\partial \ln a}\right)_{m}=-\frac{2 \Lambda}{4+c_{T}}
$$

The partial derivative $(\partial \ln T(a, m) / \partial \ln m)_{a}$ is obtained by partial differentiation of Eq. (B1) with a fixed $a$ :

$$
c_{\star}=4\left(\frac{\partial T}{\partial m}\right)_{a}+\left(\frac{\partial \ln \bar{C}_{\mathrm{d}}}{\partial \ln m}\right)_{a},
$$

where

$$
\left(\frac{\partial \ln \bar{C}_{\mathrm{d}}}{\partial \ln m}\right)_{a}=\left(\frac{\partial \ln \bar{C}_{\mathrm{d}}}{\partial \ln T}\right)_{m}\left(\frac{\partial \ln T}{\partial \ln m}\right)_{a}+\left(\frac{\partial \ln \bar{C}_{\mathrm{d}}}{\partial \ln m}\right)_{T}=c_{T}\left(\frac{\partial \ln T}{\partial \ln m}\right)_{a}+c_{\mathrm{d}} .
$$

Thus,

$$
\left(\frac{\partial \ln T}{\partial \ln m}\right)_{a}=\frac{c_{\star}-c_{\mathrm{d}}}{4+c_{T}}
$$

\section{DERIVATION OF $d a /\left.d t\right|_{\mathrm{II}}$}

The orbital-averaged drift rate $\langle d a / d t\rangle$ equals the drift rate $d a / d t$ at $r=a$ if the terms of the order of $e^{2}$ and higher are negligibly small. From Eq. (28), the drift rate in Stage II is given by

$$
\frac{d a}{d t}=\eta \frac{\beta_{\text {total }}}{1-\beta_{\text {total }}} \frac{a}{m} A P_{\mathrm{v}} \sqrt{\frac{\mu m_{\mathrm{u}}}{2 \pi k T}}-\frac{2 G M_{\star} \beta_{\mathrm{PR}}}{c a},
$$


where

$$
-\frac{d m}{d t}=A P_{\mathrm{v}} \sqrt{\frac{\mu m_{\mathrm{u}}}{2 \pi k T}}
$$

is the mass sublimation rate given by Eq. (12). In the zeroth approximation, $d a / d t=0$.

To obtain $d a / d t$ in the first approximation, we differentiate both sides of Eq. (C1) with respect to time $t$ :

$$
\begin{aligned}
\frac{d^{2} a}{d t^{2}}= & \eta \frac{\beta_{\text {total }}}{1-\beta_{\text {total }}} \frac{a}{m} \sqrt{\frac{\mu m_{\mathrm{u}}}{2 \pi k T}} A P_{\mathrm{v}} \\
\times & \left\{\frac{d \ln m}{d t}\left[\frac{d \ln \eta}{d \ln m}-\frac{\eta}{1-\beta_{\text {total }}}-1+\zeta_{A}+\left(\frac{d \ln P_{\mathrm{v}}}{d \ln T}-\frac{1}{2}\right)\left(\frac{\partial \ln T}{\partial \ln m}\right)_{a}\right]\right. \\
& \left.+\frac{d \ln a}{d t}\left[1+\left(\frac{d \ln P_{\mathrm{v}}}{d \ln T}-\frac{1}{2}\right)\left(\frac{\partial \ln T}{\partial \ln a}\right)_{m}\right]\right\} \\
- & \frac{2 G M_{\star} \beta_{\mathrm{PR}}}{c a}\left(-\eta_{\mathrm{PR}} \frac{d \ln m}{d t}-\frac{d \ln a}{d t}\right)
\end{aligned}
$$

where $\zeta_{A}=d \ln A / d \ln m$. The factors of the two terms outside the brackets on the r.h.s. are the same as the terms appearing on the r.h.s. of Eq. (C1), and can be set equal by using the result of the zeroth approximation $d a / d t=0$ to obtain $d a / d t$ in the first approximation. In addition, $d^{2} a / d t^{2}$ in the 1.h.s. of Eq. (C3) is also approximately zero. Then, we obtain

$$
\frac{d \ln a}{d t}=-\frac{1}{g_{\mathrm{m}}(T, m)} \frac{d \ln m}{d t}
$$

with

$$
g_{\mathrm{m}}(T, m)=\frac{\left(\frac{d \ln P_{\mathrm{v}}}{d \ln T}-\frac{1}{2}\right)\left(\frac{\partial \ln T}{\partial \ln a}\right)_{m}+2}{\frac{d \ln \eta}{d \ln m}-\frac{\eta}{1-\beta_{\text {total }}}-1+\zeta_{A}+\left(\frac{d \ln P_{\mathrm{v}}}{d \ln T}-\frac{1}{2}\right)\left(\frac{\partial \ln T}{\partial \ln m}\right)_{a}+\eta_{\mathrm{PR}}}
$$

from Eq. (C3). Substituting Eqs. (B12) and (B9) into Eq. (C5), we obtain Eq. (47). By approximating $d a / d t=0$ in Eq. (C1), Eq. (C4) is written as

$$
\frac{d a}{d t}=\frac{2 G M_{\star} \beta_{\mathrm{PR}}\left(1-\beta_{\text {total }}\right)}{\operatorname{ca\eta } \beta_{\text {total }} g_{\mathrm{m}}(T, m)}
$$


with the use of Eq. (C2). This is identical to $d a /\left.d t\right|_{\text {II }}$ given by Eq. (45). 


\section{REFERENCES}

Allen, C. W. 1976. Astrophysical Quantities. London: Athlone (3rd edition), 1976.

Belton, M. J. S. 1966. Dynamics of interplanetary dust. Science 151, 35-44.

Burns, J. A., Lamy, P. L., Soter, S. 1979. Radiation forces on small particles in the solar system. Icarus 40, 1-48.

Gustafson, B. A. S. 1994. Physics of zodiacal dust. Annual Review of Earth and Planetary Sciences 22, 553-595.

Kimura, H., Ishimoto, H., Mukai, T. 1997. A study on solar dust ring formation based on fractal dust models. Astronomy and Astrophysics 326, 263-270.

Kobayashi, H., Watanabe, S., Kimura, H., Yamamoto, T. 2008. Dust ring formation due to ice sublimation of radially drifting dust particles under the Poynting-Robertson effect in debris disks. Icarus 195, 871-881.

Köhler, M.,Minato, T., Kimura, H., Mann, I. 2007. Radiation pressure force acting on cometary aggregates. Advances in Space Research 40, 266-271.

Mann, I., Krivov, A., Kimura, H. 2000. Dust cloud near the Sun. Icarus 146, 568-582.

Minato, T., Köhler, M., Kimura, H., Mann, I., Yamamoto, T. 2004. Momentum Transfer to Interplanetary dust from the solar wind. Astronomy and Astrophysics 424, L13-L16. 
Minato, T., Köhler, M., Kimura, H., Mann, I., Yamamoto, T. 2006. Momentum transfer to fluffy dust aggregates from stellar winds. Astronomy and Astrophysics 452, 701-707.

Mukai, T., Yamamoto, T. 1979. A model of the circumsolar dust cloud. Publications of the Astronomical Society of Japan 31, 585-595.

Mukai, T., Ishimoto, H., Kozasa, T., Blum, J., Greenberg, J. M. 1992. Radiation pressure forces of fluffy porous grains. Astronomy and Astrophysics 262, 315-320.

Wyatt, S. P., Whipple, F. L. 1950. The Poynting-Robertson effect on meteor orbits. Astrophysical Journal 111, 134-141.

This manuscript was prepared with the AAS LATEX macros v5.0. 
Table 1: Material parameters: $\rho$ is the material density, $\mu$ is the mean molecular weight, $H$ is the latent heat of sublimation, and $P_{0}$ is the saturated vapor pressure in the limit of high temperature.

\begin{tabular}{ccccc}
\hline & $\rho\left[\mathrm{g} \mathrm{cm}^{-3}\right]$ & $\mu$ & $H\left[\mathrm{erg} \mathrm{g}^{-1}\right]$ & $P_{0}\left[\mathrm{dyn} \mathrm{cm}^{-2}\right]$ \\
\hline silicate & 2.37 & 67.0 & $7.12 \times 10^{10}$ & $1.07 \times 10^{14}$ \\
carbon & 1.95 & 12.0 & $7.27 \times 10^{11}$ & $4.31 \times 10^{16}$ \\
\hline
\end{tabular}

Reference Kimura et al. (1997)

Table 2: The sublimation distance $a_{\text {sub }}$, temperature $T_{\text {sub }}$ and the number-density enhancement factor $f_{\mathrm{N}}$ around each star.

\begin{tabular}{c||ccc|ccc|ccc}
\hline \multicolumn{1}{c||}{} & \multicolumn{3}{c|}{ Sun $^{a}$} & \multicolumn{3}{c|}{$\beta$ Pic $^{b}$} & \multicolumn{3}{c}{ Vega $^{c}$} \\
\cline { 2 - 9 } & icy & silicate & carbon & icy & silicate & carbon & icy & silicate & carbon \\
\hline$a_{\text {sub }}$ & $22 \mathrm{AU}$ & $4.9 R_{\odot}$ & $3.6 R_{\odot}$ & $31 \mathrm{AU}$ & $29 R_{\odot}$ & $11 R_{\odot}$ & $40 \mathrm{AU}$ & $73 R_{\odot}$ & $24 R_{\odot}$ \\
$T_{\text {sub }}$ & $1.0 \times 10^{2}$ & $1.2 \times 10^{3}$ & $2.1 \times 10^{3}$ & $1.0 \times 10^{2}$ & $1.2 \times 10^{3}$ & $2.0 \times 10^{3}$ & $1.1 \times 10^{2}$ & $1.2 \times 10^{3}$ & $2.0 \times 10^{3}$ \\
$f_{N}$ & 2.7 & 3.3 & 9.2 & 2.2 & 6.5 & 27 & 3.4 & 7.3 & 19 \\
\hline
\end{tabular}

${ }^{\mathrm{a}} L_{\star}=L_{\odot}, M_{\star}=M_{\odot}, T_{\star}=5780 \mathrm{~K}$

b $L_{\star}=8.7 L_{\odot}, M_{\star}=1.75 M_{\odot}, T_{\star}=8200 \mathrm{~K}$

${ }^{\mathrm{c}} L_{\star}=35 L_{\odot}, M_{\star}=2.3 M_{\odot}, T_{\star}=9988 \mathrm{~K}$

${ }^{\dagger}$ Icy dust particles that contain of both ice and organics with set of the ice volume fraction of 0.9. 
Fig. 1 - Orbital eccentricity and radius of a silicate dust particle as a function of semimajor axis of the particle. Initial semimajor axis, eccentricity, and radius are $10 R_{\odot}$, $10^{-4}$, and $1 \mu \mathrm{m}$, respectively. The solid lines show the orbital integration through the numerical integration of Eqs. (23)-(25). The dotted lines indicate Eqs. (37) and (44) from $\left(a_{0}, s_{0}\right)$ to $\left(a_{1}, s_{1}\right)$ and Eqs. (53) and (58) from $\left(a_{1}, s_{1}\right)$, where $a_{1}$ and $s_{1}$ are at the intersection between Eqs. (44) and (53) and the $a_{1}, s_{1}$, and $e_{1}$ are shown by the crosses.

Fig. 2 - Same as 1, but for carbon.

Fig. 3 - The number density $N$, for silicate divided by $r_{0} N_{0} / r$ as a function of distance from Sun in the solar radius, assuming a constant dust flux due to the P-R drag from $6 R_{\odot}$. The solid line indicates the numerical simulation. The circle represents the number density and the distance at the peak, respectively, given by Eq. (68) and Eq. (53) for $m_{0 \min }$.

Fig. 4 - Same as Fig. 3, but for carbon.

Fig. 5 - The number-density enhancement factor $f_{N}$ and the effective-optical-depth enhancement factor $f_{\tau_{\mathrm{eff}}}$ for silicate dust calculated from Eqs. (68) and (69), assuming that dust particles with $\beta=0.1-0.5$ radially drift into the sublimation zone by the P-R drag (top panel) and the sublimation distance (bottom panel) given by Eq. (53) for the silicate dust with $\beta=0.5$. For $L_{\star} \lesssim 0.8 L_{\odot}$ (in the grey zone), the maximum value of $\beta$ is less than 0.5 . Characters on the top of the figure indicate spectral classes of central stars.

Fig. 6 - Same as Fig. 5, but for carbon. 


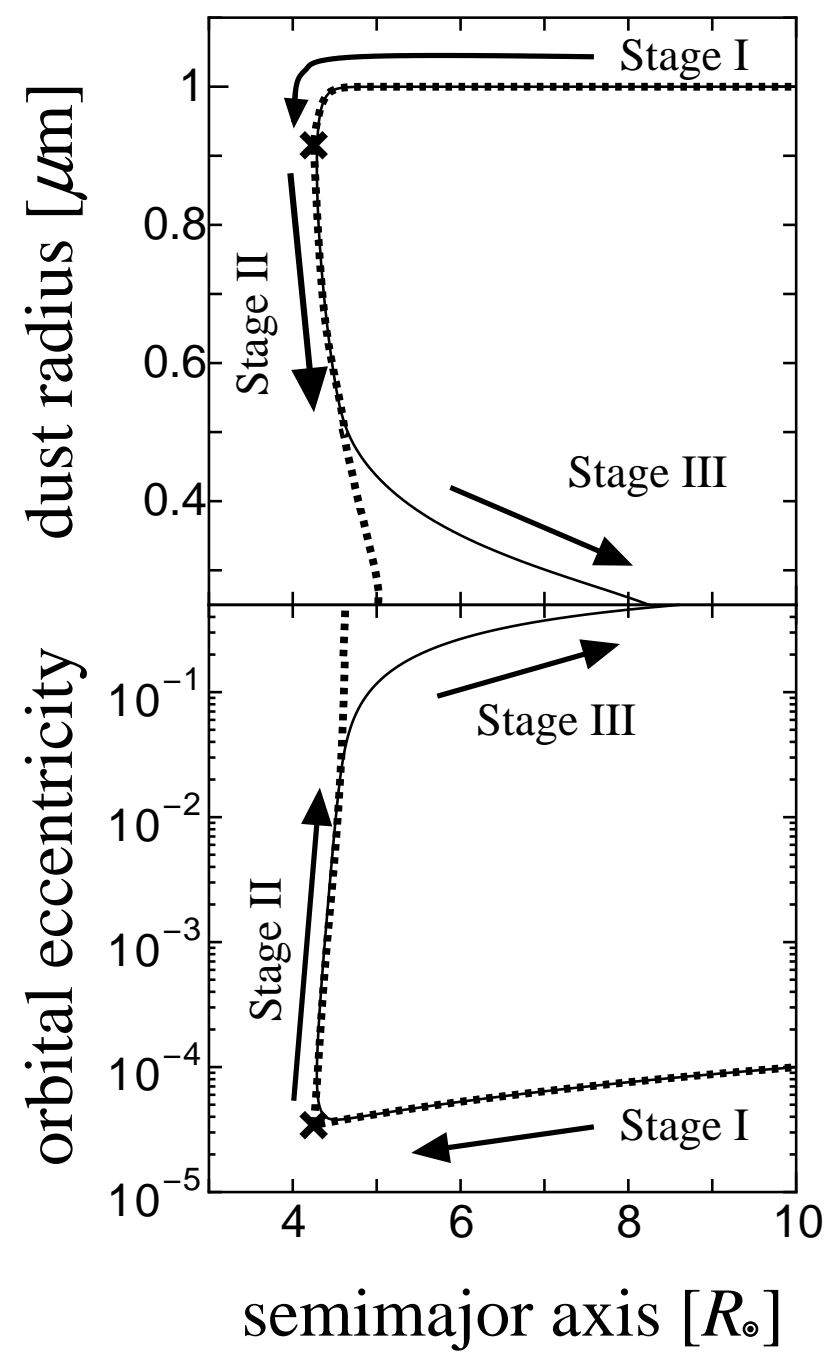

Fig. 1. - Orbital eccentricity and radius of a silicate dust particle as a function of semimajor axis of the particle. Initial semimajor axis, eccentricity, and radius are $10 R_{\odot}, 10^{-4}$, and $1 \mu \mathrm{m}$, respectively. The solid lines show the orbital integration through the numerical integration of Eqs. (23)-(25). The dotted lines indicate Eqs. (37) and (44) from $\left(a_{0}, s_{0}\right)$ to $\left(a_{1}, s_{1}\right)$ and Eqs. (53) and (58) from $\left(a_{1}, s_{1}\right)$, where $a_{1}$ and $s_{1}$ are at the intersection between Eqs. (44) and (53) and the $a_{1}, s_{1}$, and $e_{1}$ are shown by the crosses. 


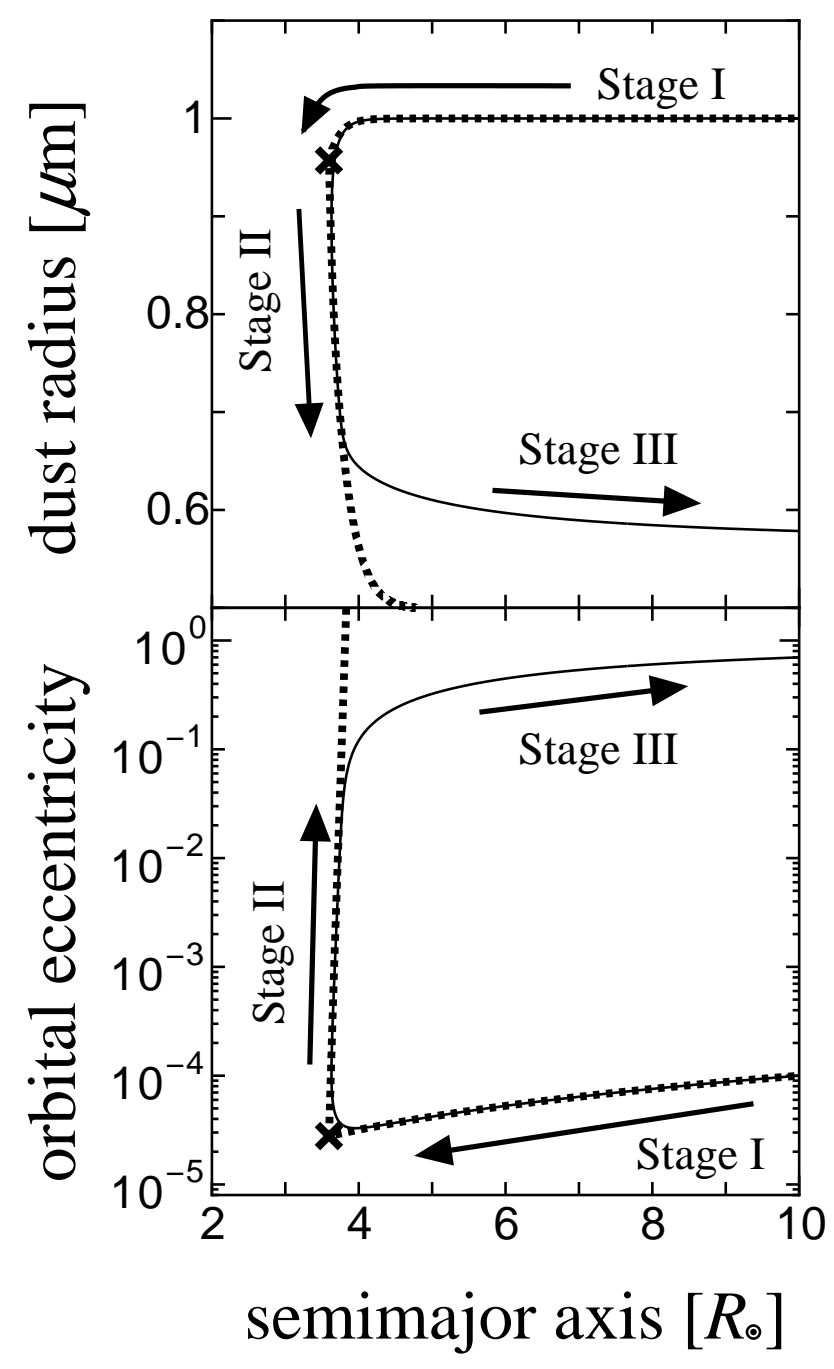

Fig. 2.- Same as 1, but for carbon. 


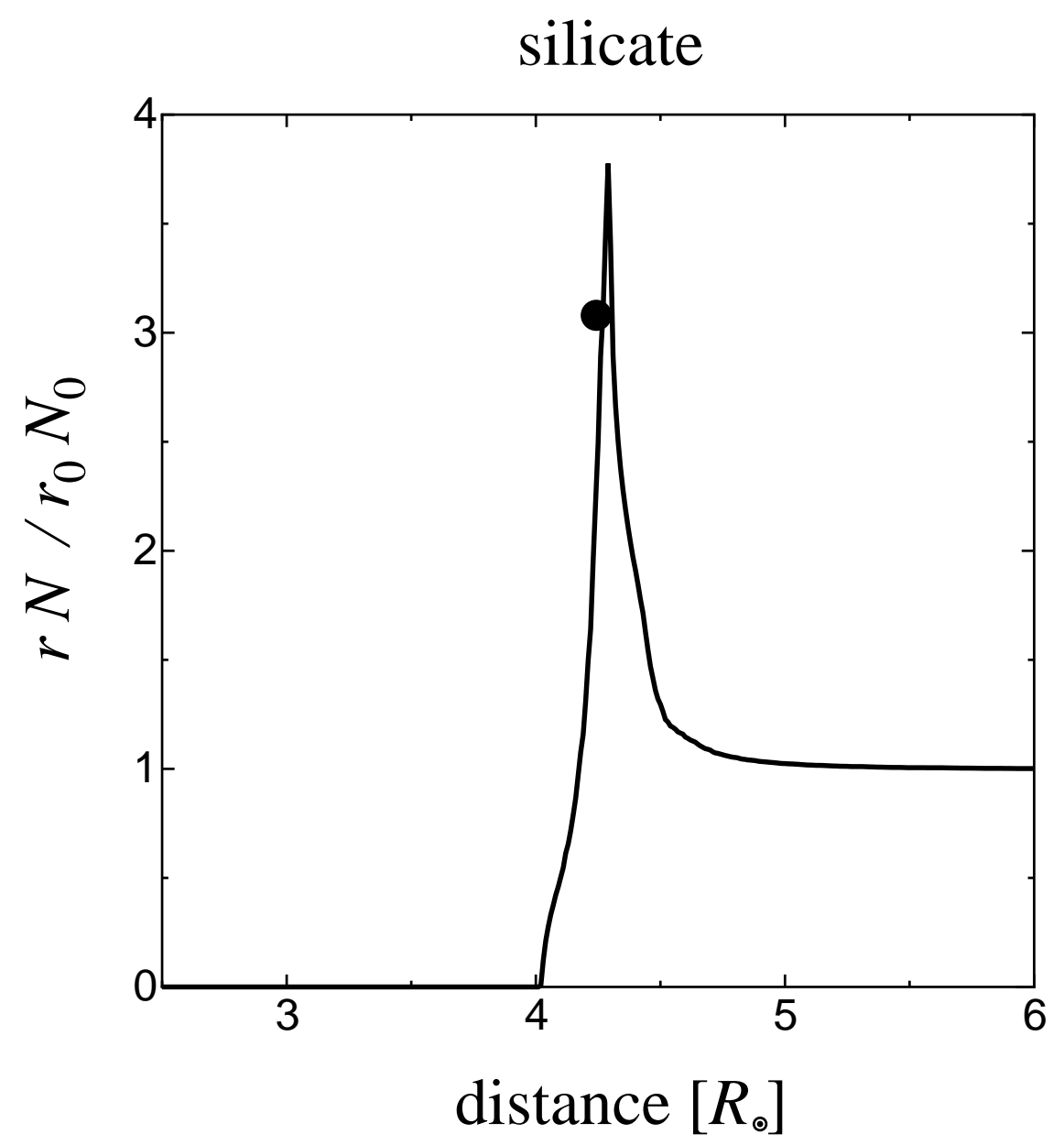

Fig. 3.- The number density $N$, for silicate divided by $r_{0} N_{0} / r$ as a function of distance from Sun in the solar radius, assuming a constant dust flux due to the P-R drag from $6 R_{\odot}$. The solid line indicates the numerical simulation. The circle represents the number density and the distance at the peak, respectively, given by Eq. (68) and Eq. (53) for $m_{0 \text { min }}$. 


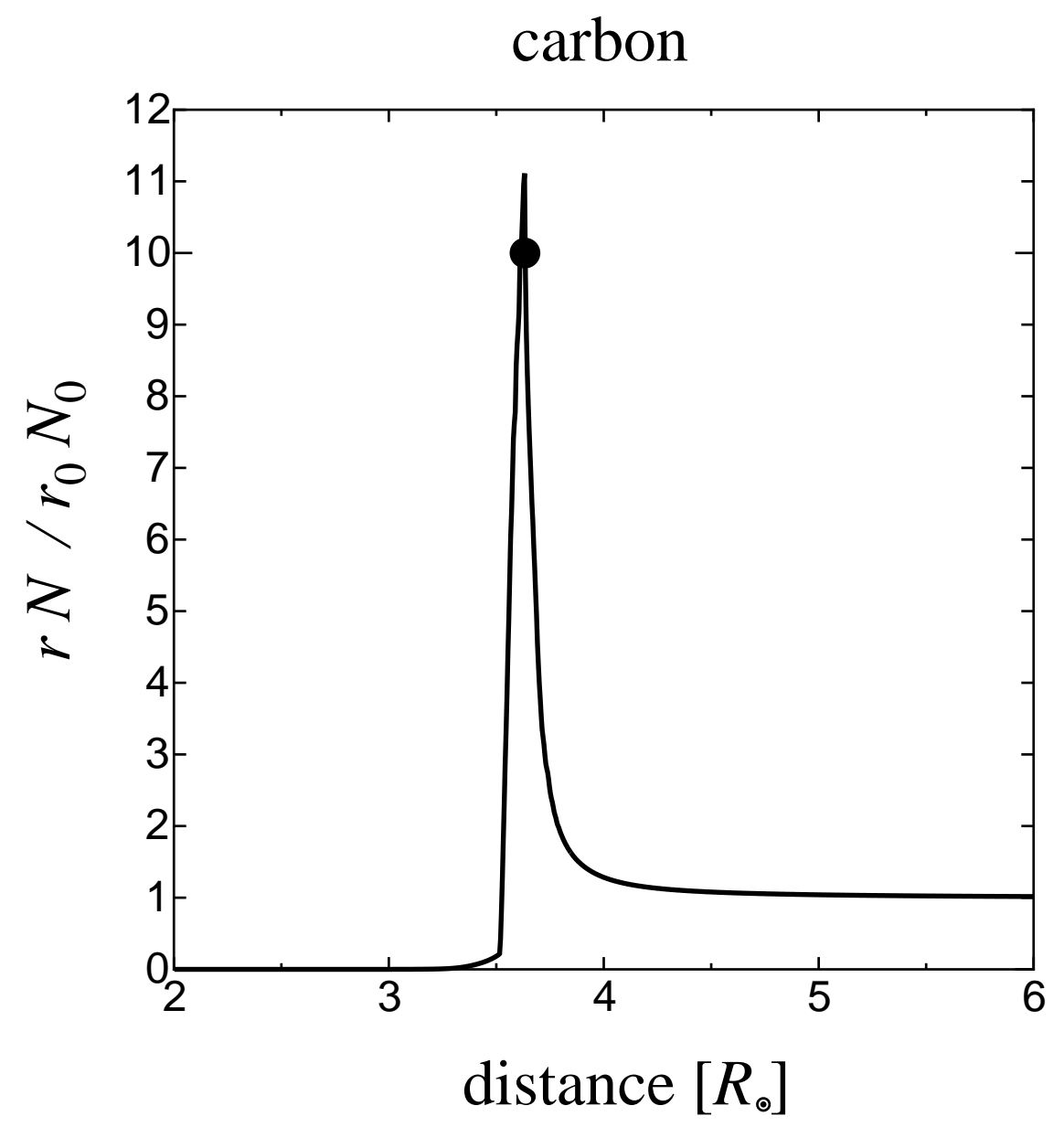

Fig. 4. - Same as Fig. 3, but for carbon. 


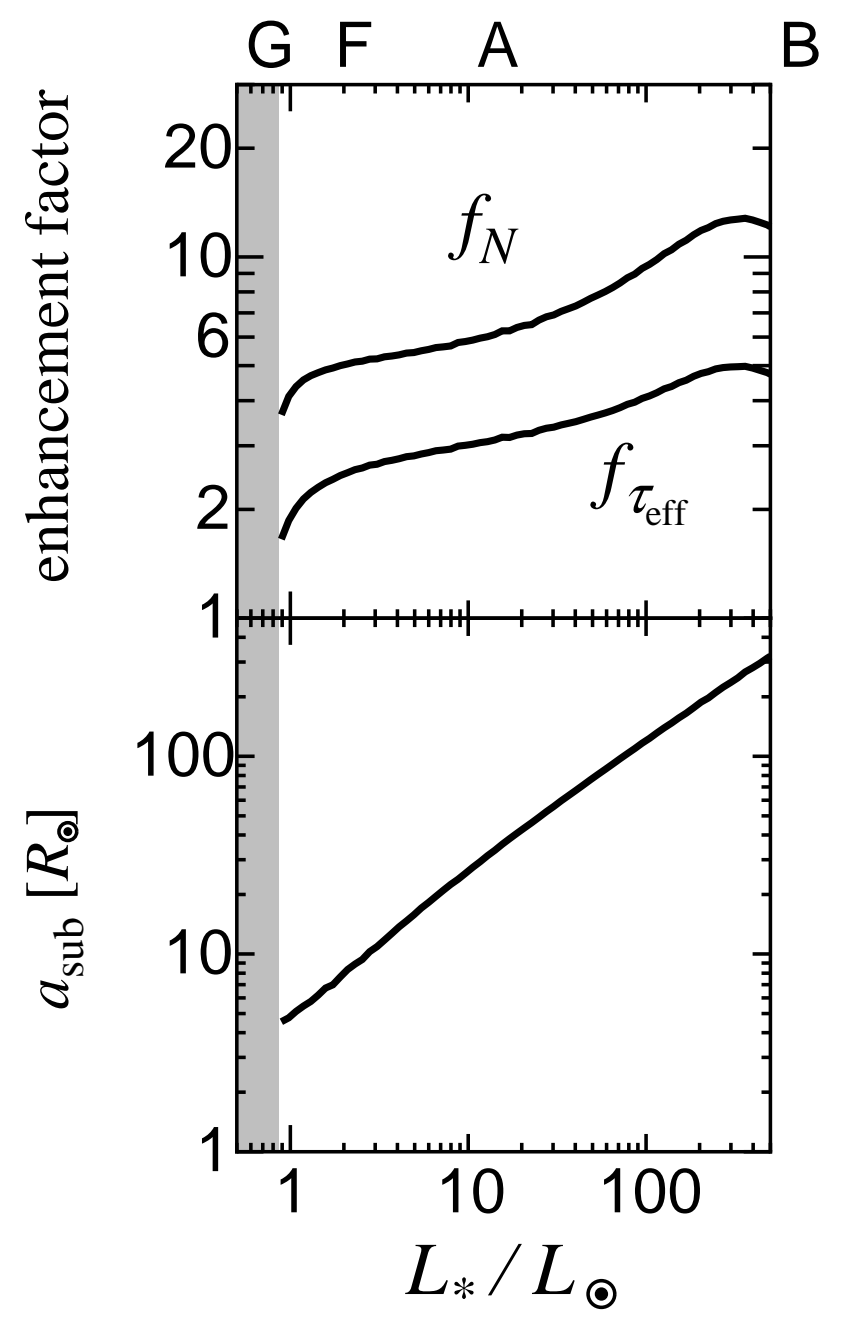

Fig. 5.- The number-density enhancement factor $f_{N}$ and the effective-optical-depth enhancement factor $f_{\tau_{\mathrm{eff}}}$ for silicate dust calculated from Eqs. (68) and (69), assuming that dust particles with $\beta=0.1-0.5$ radially drift into the sublimation zone by the P-R drag (top panel) and the sublimation distance (bottom panel) given by Eq. (53) for the silicate dust with $\beta=0.5$. For $L_{\star} \lesssim 0.8 L_{\odot}$ (in the grey zone), the maximum value of $\beta$ is less than 0.5 . Characters on the top of the figure indicate spectral classes of central stars. 


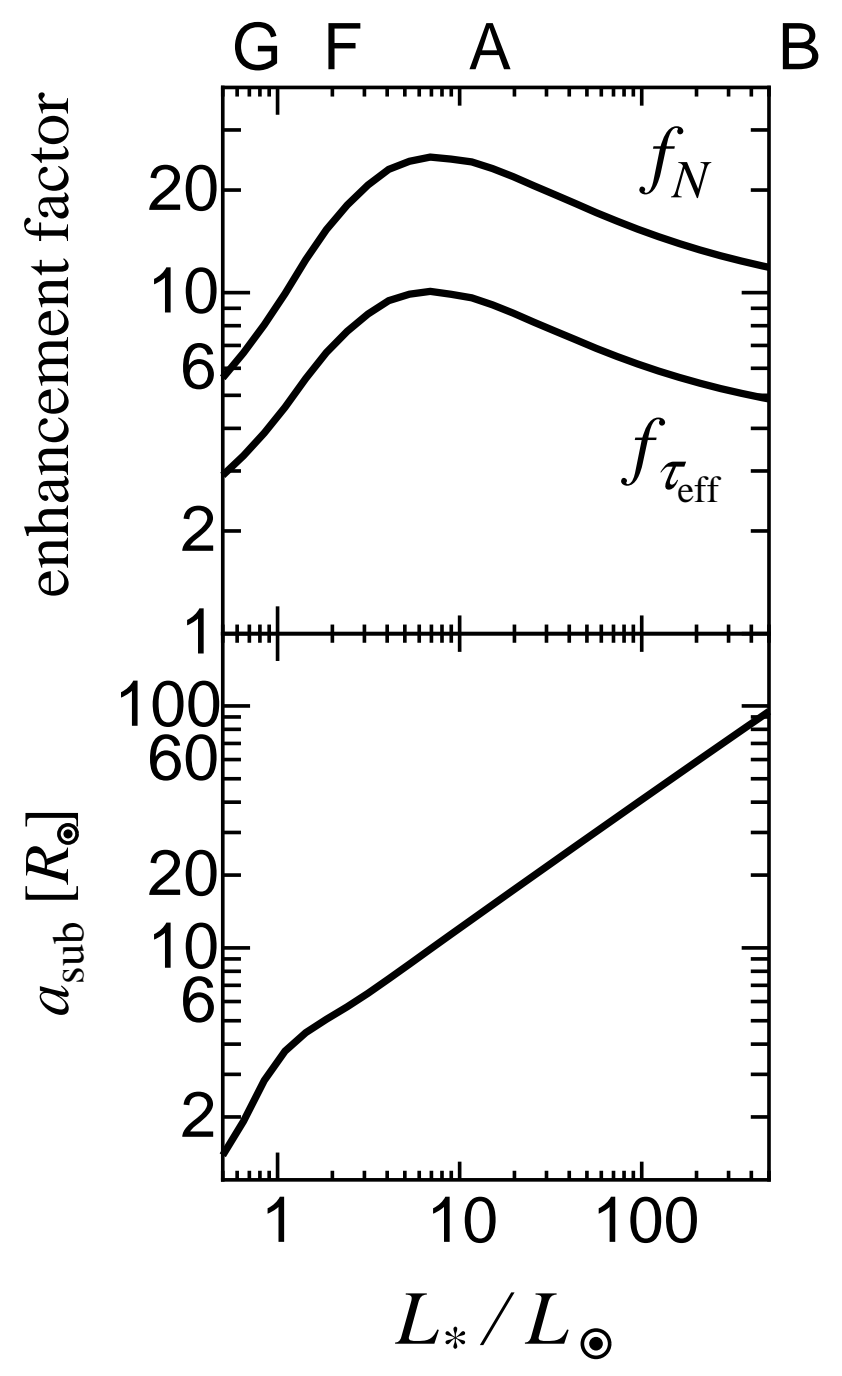

Fig. 6.- Same as Fig. 5, but for carbon. 